\title{
$N \Omega$ interaction: Meson exchanges, inelastic channels, and quasibound state
}

\author{
Takayasu Sekihara, ${ }^{1, *}$ Yuki Kamiya, ${ }^{2, \dagger}$ and Tetsuo Hyodo ${ }^{2, \dagger}$ \\ ${ }^{1}$ Advanced Science Research Center, Japan Atomic Energy Agency, Shirakata, Tokai, Ibaraki 319-1195, Japan \\ ${ }^{2}$ Yukawa Institute for Theoretical Physics, Kyoto University, Kyoto 606-8502, Japan
}

(Received 15 May 2018; published 18 July 2018)

\begin{abstract}
Based on a baryon-baryon interaction model with meson exchanges, we investigate the origin of the strong attraction in the $N \Omega\left({ }^{5} S_{2}\right)$ interaction, which was indicated by recent lattice QCD simulations. The long-range part of the potential is constructed by the conventional mechanisms, the exchanges of the $\eta$ meson and of the correlated two mesons in the scalar-isoscalar channel, denoted by " $\sigma$ " in the literature. The short-range part is represented by the contact interaction. We find that the meson exchanges do not provide sufficient attraction. This means that most of the attraction is attributed to the short-range contact interaction. We then evaluate the effect of the coupled channels to the $N \Omega\left({ }^{5} S_{2}\right)$ interaction. We find that, while the $D$-wave mixing of the $N \Omega$ channel is negligible, the inelastic $\Lambda \Xi, \Sigma \Xi$, and $\Lambda \Xi(1530)$ channels via the $K$ meson exchange give the attraction of the $N \Omega\left({ }^{5} S_{2}\right)$ interaction to the same level with the elastic meson exchanges. Although the elimination of these channels induces the energy dependence of the single-channel $N \Omega$ interaction, this effect is not significant. With the present model parameters fit to reproduce the scattering length of the HAL QCD result of the nearly physical quark masses, we obtain the $N \Omega\left({ }^{5} S_{2}\right)$ quasibound state with its eigenenergy $2611.3-0.7 i \mathrm{MeV}$, which corresponds to the binding energy $0.1 \mathrm{MeV}$ and width $1.5 \mathrm{MeV}$ for the decay to the $\Lambda \Xi$ and $\Sigma \Xi$ channels. From the analysis of the spatial structure and the compositeness, the quasibound state is shown to be the molecular state of $N \Omega$. We also construct an equivalent local potential for the $N \Omega\left({ }^{5} S_{2}\right)$ system which is useful for various applications.
\end{abstract}

DOI: 10.1103/PhysRevC.98.015205

\section{INTRODUCTION}

Existence and properties of dibaryons have been one of the major topics in hadron physics. Here dibaryons stand for states of baryon number $B=2$ generated by strong interactions regardless of their structure: compact hexaquarks, baryonbaryon, and meson-baryon-baryon molecules, etc. So far, there is only the single well-established dibaryon state, the deuteron, which is a proton-neutron molecule [1]. Because various different mechanisms in strong interactions are expected to generate dibaryons, the study of dibaryons helps to understand the underlying theory of strong interactions, quantum chromodynamics (QCD). For instance, compact hexaquarks are closely related to the mechanism of quark confinement and correlation of quarks inside hadrons. Hadronic molecules serve as a valuable clue to investigate the hadron-hadron interactions, which lead to novel few-body systems bound by hadronic interactions.

Historically, dibaryons were first discussed in theoretical studies. In the early stage, dibaryons analogous to the deuteron

\footnotetext{
*sekihara@post.j-parc.jp

†yuki.kamiya@yukawa.kyoto-u.ac.jp

${ }^{\ddagger}$ hyodo@yukawa.kyoto-u.ac.jp
}

Published by the American Physical Society under the terms of the Creative Commons Attribution 4.0 International license. Further distribution of this work must maintain attribution to the author $(s)$ and the published article's title, journal citation, and DOI. Funded by $S C O A P^{3}$. were predicted in Ref. [2] by combining the nucleon $(N)$ and the $\Delta$ resonance. The $H$ dibaryon was predicted as a compact hexaquark owing to the strongly attractive colormagnetic interaction between quarks [3], which stimulated various experimental searches for such dibaryons (see review [4]). As an example of the dibaryon with meson-baryon-baryon structure, the $\bar{K} N N$ state was predicted [5], motivated by the strong attraction between the antikaon $(\bar{K})$ and nucleon [6] (see reviews [7,8]). Then, recent remarkable progress in experiments and in lattice QCD simulations enables us to examine these theoretical predictions on dibaryons. For example, the WASA-at-COSY collaboration recently reported the $d^{*}(2380)$ in quantum numbers $\left(J^{P}, I\right)=\left(3^{+}, 0\right)$ [9-11], which may correspond to the isoscalar $\Delta \Delta$ bound state predicted in Ref. [2]. Some hints about the $H$ dibaryon come from lattice QCD simulations [12-19]. In particular, the baryon-baryon interaction in the HAL QCD method with nearly physical quark masses [18,19] implies the existence of a resonance around the $N \Xi$ threshold. Lattice QCD analyses with the nearly physical quark masses are further suggesting new dibaryons such as the $S$-wave $\Omega \Omega$ bound state in $J^{P}=0^{+}$[20]. The J-PARC E15 experiment observed a peak structure which can be interpreted as a signal of the $\bar{K} N N$ quasibound state $[21,22]$.

In this study, we focus on yet another dibaryon system, the $N \Omega$ state. This system was predicted to be bound in quarkmodel calculations [23-26], and further studies within quark models are found in Refs. [27,28]. Remarkably, the repulsive core is expected to be absent in the elastic $N \Omega$ potential, in contrast with the nuclear force, because the quark flavors in $N$ are completely different from those in $\Omega$ and hence the Pauli 
TABLE I. Baryon-baryon channels coupling to $N \Omega$ and their threshold energies.

\begin{tabular}{lc}
\hline \hline Channel & Threshold [MeV] \\
\hline$\Lambda \Xi$ & 2434 \\
$\Sigma \Xi$ & 2511 \\
$N \Omega$ & 2611 \\
$\Lambda \Xi(1530)=\Lambda \Xi^{*}$ & 2649 \\
$\Sigma(1385) \Xi=\Sigma^{*} \Xi$ & 2703 \\
$\Sigma \Xi(1530)=\Sigma \Xi^{*}$ & 2727 \\
$\Sigma(1385) \Xi(1530)=\Sigma^{*} \Xi^{*}$ & 2918 \\
\hline \hline
\end{tabular}

exclusion principle does not work. The absence of the repulsive core is advantageous to generate a possible dibaryon state in the $N \Omega$ system. The $N \Omega$ interaction in the ${ }^{5} S_{2}$ channel was recently obtained in the HAL QCD analyses of the lattice QCD data, where ${ }^{2 S+1} L_{J}$ denotes the state with spin $S, L$ wave, and total angular momentum $J$ of the $N \Omega$ system. Interestingly, the results of the HAL QCD analyses suggested a strongly attractive potential in the $N \Omega\left({ }^{5} S_{2}\right)$ channel without repulsive core which supports a bound state [29-31]. Although there are lower-energy baryon-baryon coupled channels $\Lambda \Xi$ and $\Sigma \Xi$, it is expected that the decay of the $N \Omega\left({ }^{5} S_{2}\right)$ quasibound state will be suppressed because couplings to these decay channels are in $D$ wave (see Table I for baryon-baryon channels coupling to the $N \Omega$ state). Stimulated by the HAL QCD results, the $N \Omega$ interaction was studied in the framework of chiral perturbation theory [32]. A method to probe this dibaryon with the correlation between $N$ and $\Omega$ in high-energy heavy-ion collisions was proposed in Ref. [33] as well.

The aim of our study is to understand the origin of the strong attraction in the $N \Omega\left({ }^{5} S_{2}\right)$ channel. For this purpose, we construct a meson exchange model for the $N \Omega$ interaction. Combining the long-range meson exchange mechanisms with the short-range interaction represented by the contact term, we can pin down the physical origin of the attractive $N \Omega$ interaction. In addition, by taking into account the coupling to the relevant baryon-baryon inelastic channels, we can further discuss the absorption processes and the energy dependence of the $N \Omega$ interaction. These effects were assumed to be small and are neglected in the HAL QCD analyses of the $N \Omega$ interaction. Finally, the attractive $N \Omega$ interaction implies the possible existence of nuclei with an $\Omega$ baryon. It is practically useful to construct a local potential equivalent to the full model, for the application to few-body calculations of $\Omega$ nuclei.

This paper is organized as follows: First, in Sec. II we formulate the $N \Omega$ interaction including the inelastic contributions

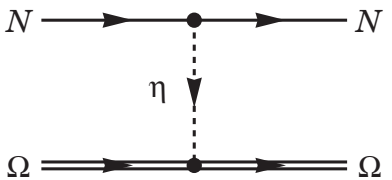

(a)

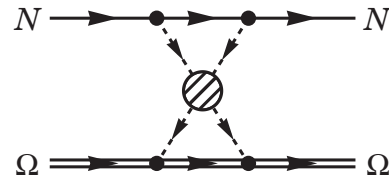

(b)

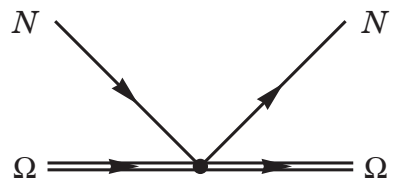

(c)

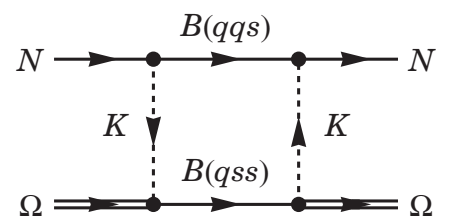

(box)

FIG. 1. Feynman diagrams for the $N \Omega$ interaction. The dashed lines represent the pseudoscalar mesons, while the solid and double lines indicate baryons. Shaded circle denotes the correlation of two mesons, and $B(q q s) B(q s s)$ represents $\Lambda \Xi, \Sigma \Xi$, and $\Lambda \Xi(1530)$. 


\section{B. Effective Lagrangians}

The vertices in Fig. 1 are constructed with the effective Lagrangians, including the pseudoscalar meson $P$, octet baryon $B$, and decuplet baryon $D$, based on flavor SU(3) symmetry.

The $P B B$ coupling is governed by the chiral Lagrangian:

$$
\begin{aligned}
\mathcal{L}= & -\frac{F}{\sqrt{2} f}\left\langle\overline{\mathcal{B}} \gamma^{\mu} \gamma_{5}\left[\partial_{\mu} \Phi, \mathcal{B}\right]\right\rangle \\
& -\frac{D}{\sqrt{2} f}\left\langle\overline{\mathcal{B}} \gamma^{\mu} \gamma_{5}\left\{\partial_{\mu} \Phi, \mathcal{B}\right\}\right\rangle,
\end{aligned}
$$

with the pseudoscalar meson and octet baryon fields $\Phi$ and $\mathcal{B}$, respectively, whose explicit forms are

$$
\begin{aligned}
& \Phi=\left(\begin{array}{ccc}
\frac{1}{\sqrt{2}} \pi^{0}+\frac{1}{\sqrt{6}} \eta & \pi^{+} & K^{+} \\
\pi^{-} & -\frac{1}{\sqrt{2}} \pi^{0}+\frac{1}{\sqrt{6}} \eta & K^{0} \\
K^{-} & \bar{K}^{0} & -\frac{2}{\sqrt{6}} \eta
\end{array}\right), \\
& \mathcal{B}=\left(\begin{array}{ccc}
\frac{1}{\sqrt{2}} \Sigma^{0}+\frac{1}{\sqrt{6}} \Lambda & \Sigma^{+} & p \\
\Sigma^{-} & -\frac{1}{\sqrt{2}} \Sigma^{0}+\frac{1}{\sqrt{6}} \Lambda & n \\
\Xi^{-} & \Xi^{0} & -\frac{2}{\sqrt{6}} \Lambda
\end{array}\right) .
\end{aligned}
$$

The meson decay constant $f$ is chosen at their physical values [34]: $f_{\pi}=92.1 \mathrm{MeV}, f_{K}=1.2 f_{\pi}$, and $f_{\eta}=1.3 f_{\pi}$. The parameters $D=0.795$ and $F=0.465$ are fixed by the weak decays of the octet baryons.

The Lagrangian for the $P B D$ coupling is

$$
\mathcal{L}=-\frac{f_{P B D}}{m_{\pi}}\left\langle\left(\bar{\Delta}_{\mu} \cdot \partial^{\mu} \Phi\right) \mathcal{B}+\text { H.c. }\right\rangle,
$$

where $m_{\pi}$ is the pion mass and the product $(\bar{\Delta} \cdot \Phi)$ represents

$$
(\bar{\Delta} \cdot \Phi)_{a b}=\epsilon_{i j a} \bar{\Delta}_{i k b} \Phi_{k j},
$$

with the decuplet baryon field $\Delta$ :

$$
\begin{aligned}
& \Delta_{111}=\Delta^{++}, \quad \Delta_{112}=\frac{1}{\sqrt{3}} \Delta^{+}, \\
& \Delta_{122}=\frac{1}{\sqrt{3}} \Delta^{0}, \quad \Delta_{222}=\Delta^{-},
\end{aligned}
$$

for $\Delta(1232)$,

$$
\Delta_{113}=\frac{1}{\sqrt{3}} \Sigma^{*+}, \quad \Delta_{123}=\frac{1}{\sqrt{6}} \Sigma^{* 0}, \quad \Delta_{223}=\frac{1}{\sqrt{3}} \Sigma^{*-},
$$

for $\Sigma(1385)$,

$$
\Delta_{133}=\frac{1}{\sqrt{3}} \Xi^{* 0}, \quad \Delta_{233}=\frac{1}{\sqrt{3}} \Xi^{*-},
$$

for $\Xi(1530)$, and

$$
\Delta_{333}=\Omega^{-},
$$

for $\Omega^{-}$. The form of $\Delta_{a b c}$ is completely symmetric under permutations of indices $a, b$, and $c=1,2,3$. The coupling constant $f_{P B D}$ is fixed as $f_{P B D}=1.8$ so as to reproduce semiquantitatively the decay widths of the decuplet baryons: with $f_{P B D}=1.8$, we obtain $\Gamma_{\triangle(1232) \rightarrow \pi N}=$ $63 \mathrm{MeV}, \Gamma_{\Sigma(1385) \rightarrow \pi \Lambda}=33 \mathrm{MeV}, \Gamma_{\Sigma(1385) \rightarrow \pi \Sigma}=5 \mathrm{MeV}$, and $\Gamma_{\Xi(1530) \rightarrow \pi \Xi}=14 \mathrm{MeV}$.
Similarly, the Lagrangian for the $P D D$ coupling is

$$
\mathcal{L}=-\frac{f_{P D D}}{m_{\pi}}\left\langle\left(\bar{\Delta}^{\mu} \cdot \gamma^{\nu} \gamma_{5} \Delta_{\mu}\right) \partial_{\nu} \Phi\right\rangle,
$$

where the product $(\bar{\Delta} \cdot \Delta)$ represents

$$
(\bar{\Delta} \cdot \Delta)_{a b}=\bar{\Delta}_{i j b} \Delta_{i j a} .
$$

The coupling constant $f_{P D D}$ is fixed from the nucleon axial charge based on the nonrelativistic SU(6) quark model [35]:

$$
\frac{g_{A}}{2}: \frac{f \times f_{P D D}}{\sqrt{2} m_{\pi}}=\frac{5}{6}: \frac{3}{2},
$$

where $g_{A} \equiv D+F=1.26$. From this analysis, we obtain $f_{P D D}=9 g_{A} m_{\pi} /(5 \sqrt{2} f)=2.09$.

Finally, we employ a spin-independent form for the contact $B D B D$ Lagrangian

$$
\mathcal{L}=c(\bar{\Omega} \Omega)(\bar{p} p+\bar{n} n),
$$

with a coupling constant $c$ as a model parameter. In general there is a spin-dependent contact $B D B D$ term as in Ref. [32], which generates difference between interactions of the $J^{P}=$ $2^{+}$and $1^{+}$channels. However, the term in Eq. (13) is sufficient in this study because we focus only on the $J^{P}=2^{+}$channel.

In the following we construct the $N \Omega$ interaction by using these effective Lagrangians, together with the empirical information of the two-meson correlation.

\section{C. $\eta$ exchange}

The $\eta$ exchange term, which is denoted $V_{\mathrm{A}}$, can be straightforwardly formulated according to Fig. 1(a). From the effective Lagrangians, we can express $V_{\mathrm{A}}$ in terms of the helicity eigenstates as

$$
\begin{aligned}
V_{\mathrm{A}}= & \frac{2 \sqrt{2}(D-3 F) f_{P D D} m_{N} m_{\Omega}}{3 f_{\eta} m_{\pi}} \frac{F(q)^{2}}{q^{2}+m_{\eta}^{2}} \bar{u}_{N}\left(\boldsymbol{p}^{\prime}, \lambda_{N}^{\prime}\right) \\
& \times \gamma_{5} u_{N}\left(\boldsymbol{p}, \lambda_{N}\right) \bar{u}_{\Omega \mu}\left(-\boldsymbol{p}^{\prime}, \lambda_{\Omega}^{\prime}\right) \gamma_{5} u_{\Omega}^{\mu}\left(-\boldsymbol{p}, \lambda_{\Omega}\right),
\end{aligned}
$$

where $\boldsymbol{p}$ and $\boldsymbol{p}^{\prime}$ are the center-of-mass relative momenta in the initial and final states, respectively, $q \equiv\left|\boldsymbol{p}-\boldsymbol{p}^{\prime}\right|$ is the momentum transfer, $m_{\eta}$ is the $\eta$ mass, and $u_{N}$ and $u_{\Omega}^{\mu}$ are the $N$ and $\Omega$ spinors, respectively (see Appendix B). The spinors depend on the helicity $\lambda$ as well as the momentum $\boldsymbol{p}$. We introduced a form factor $F(q)$ of a monopole type:

$$
F(q)=\frac{\Lambda^{2}}{\Lambda^{2}+q^{2}},
$$

with cutoff $\Lambda$. In the calculation of $V_{\mathrm{A}}$ we used the relations

$$
\begin{gathered}
\bar{u}_{N}\left(\not p_{N}^{\prime}-\not p_{N}\right) \gamma_{5} u_{N}=2 m_{N} \bar{u}_{N} \gamma_{5} u_{N}, \\
\bar{u}_{\Omega \mu}\left(\not p_{\Omega}^{\prime}-\not p_{\Omega}\right) \gamma_{5} u_{\Omega}^{\mu}=2 m_{\Omega} \bar{u}_{\Omega \mu} \gamma_{5} u_{\Omega}^{\mu},
\end{gathered}
$$

where $m_{N}$ and $m_{\Omega}$ are the $N$ and $\Omega$ masses, respectively.

\section{Correlated two-meson exchange}

To formulate the correlated two-meson exchange term $V_{\mathrm{B}}$, we need some consideration. In this study we start with a general form of the interaction constructed as a linear 
combination of the so-called kinematic covariants $\mathcal{O}_{a}^{(N)}$ and $\mathcal{O}_{a \mu \nu}^{(\Omega)}[36,37]:$

$$
\begin{aligned}
V_{\mathrm{B}}= & \sum_{a} \mathcal{V}_{a}(t) \bar{u}_{N}\left(\boldsymbol{p}^{\prime}, \lambda_{N}\right) \mathcal{O}_{a}^{(N)} u_{N}\left(\boldsymbol{p}, \lambda_{N}\right) \\
& \times \bar{u}_{\Omega}^{\mu}\left(-\boldsymbol{p}^{\prime}, \lambda_{\Omega}\right) \mathcal{O}_{a \mu \nu}^{(\Omega)} u_{\Omega}^{\nu}\left(-\boldsymbol{p}, \lambda_{\Omega}\right),
\end{aligned}
$$

where the coefficients $\mathcal{V}_{a}$ are Lorentz-invariant amplitudes as functions of the Mandelstam variable $t$ and $a$ specifies types of $\left(\mathcal{O}_{a}^{(N)}, \mathcal{O}_{a \mu \nu}^{(\Omega)}\right)$. The kinematic covariants are built up from the Dirac matrices and the momenta in such a way that their bilinear spinor representations are Lorentz invariant. Then, because the correlated two mesons in Fig. 1 are in the scalar channel, we have only two independent types of $\left(\mathcal{O}_{a}^{(N)}, \mathcal{O}_{a \mu \nu}^{(\Omega)}\right)$, for which we take

$$
\begin{aligned}
& a=\mathrm{S}: \mathcal{O}_{\mathrm{S}}^{(N)}=1, \quad \mathcal{O}_{\mathrm{S} \mu \nu}^{(\Omega)}=g_{\mu \nu}, \\
& a=2 \mathrm{M}: \mathcal{O}_{2 \mathrm{M}}^{(N)}=1, \quad \mathcal{O}_{2 \mathrm{M} \mu \nu}^{(\Omega)}=\frac{q_{\mu} q_{\nu}}{m_{\Omega}^{2}},
\end{aligned}
$$

where $q^{\mu}$ is the four-momentum transfer. Therefore, making the three-dimensional reduction and introducing a phenomenological form factor, we can express $V_{\mathrm{B}}$ as

$$
\begin{aligned}
V_{\mathrm{B}}= & F(q)^{2} \bar{u}_{N}\left(\boldsymbol{p}^{\prime}, \lambda_{N}^{\prime}\right) u_{N}\left(\boldsymbol{p}, \lambda_{N}\right) \\
& \times\left[\mathcal{V}_{\mathrm{S}}(t) \bar{u}_{\Omega \mu}\left(-\boldsymbol{p}^{\prime}, \lambda_{\Omega}^{\prime}\right) u_{\Omega}^{\mu}\left(-\boldsymbol{p}, \lambda_{\Omega}\right)\right. \\
& \left.+\mathcal{V}_{2 \mathrm{M}}(t) \frac{q_{\mu} q_{v}}{m_{\Omega}^{2}} \bar{u}_{\Omega}^{\mu}\left(-\boldsymbol{p}^{\prime}, \lambda_{\Omega}^{\prime}\right) u_{\Omega}^{v}\left(-\boldsymbol{p}, \lambda_{\Omega}\right)\right],
\end{aligned}
$$

where $t=-\left|\boldsymbol{p}-\boldsymbol{p}^{\prime}\right|^{2}$ and $q^{\mu}=\left(0, \boldsymbol{p}-\boldsymbol{p}^{\prime}\right)$. We use the monopole-type form factor in Eq. (15) with the same cutoff $\Lambda$.

Now our task is to evaluate the coefficients $\mathcal{V}_{\mathrm{S}}$ and $\mathcal{V}_{2 \mathrm{M}}$, which govern the interaction strength. They are calculated with the dispersion relation for the scattering amplitude, as done in, e.g., Refs. [36,37]. In the $N \Omega \rightarrow N \Omega$ reaction, $\mathcal{V}_{\mathrm{S}, 2 \mathrm{M}}(t)$ as a function of $t$ is analytic except for some resonance poles and branch cuts along the real $t$ line: the unitarity cut running from $4 m_{\pi}^{2}$ to $\infty$ and the left-hand cuts. Therefore, neglecting the latter one, which is irrelevant to the correlated two-meson exchange, we may consider the dispersion relation in a general form

$$
\mathcal{V}_{\mathrm{S}, 2 \mathrm{M}}(t)=\frac{1}{\pi} \int_{4 m_{\pi}^{2}}^{t_{c}} d t^{\prime} \frac{\operatorname{Im} \mathcal{V}_{\mathrm{S}, 2 \mathrm{M}}\left(t^{\prime}\right)}{t^{\prime}-t},
$$

where we introduced a cutoff $t_{c}$ instead of infinity. Equation (22) means that, to calculate the $N \Omega$ interaction with the correlated two-meson exchange taking place in the region

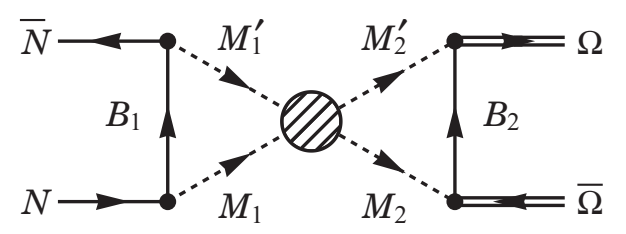

FIG. 2. Feynman diagram for the $N \bar{N} \rightarrow \Omega \bar{\Omega}$ reaction. Particles in the intermediate states are listed in Table II. Shaded circle denotes the correlation of two mesons.
TABLE II. Particles in the intermediate states of the diagram in Fig. 2.

\begin{tabular}{lccccc}
\hline \hline$B_{1}$ & $M_{1}$ & $M_{1}^{\prime}$ & $B_{2}$ & $M_{2}$ & $M_{2}^{\prime}$ \\
\hline$N$ & $\pi$ & $\pi$ & $\Omega$ & $\eta$ & $\eta$ \\
$N$ & $\eta$ & $\eta$ & $\Xi$ & $K$ & $\bar{K}$ \\
$\Delta$ & $\pi$ & $\pi$ & $\Xi^{*}$ & $K$ & $\bar{K}$ \\
$\Lambda$ & $K$ & $\bar{K}$ & & & \\
$\Sigma$ & $K$ & $\bar{K}$ & & & \\
$\Sigma^{*}$ & $K$ & $\bar{K}$ & & & \\
\hline \hline
\end{tabular}

$t<0$, we may consider the same amplitude but in $t>4 m_{\pi}^{2}$, which can be achieved in the $N \bar{N} \rightarrow \Omega \bar{\Omega}$ reaction as shown in Fig. 2.

In this study we formulate the $N \bar{N} \rightarrow \Omega \bar{\Omega}$ scattering amplitude by considering the intermediate states listed in Table II. The scattering amplitude of the two pseudoscalar mesons in the scalar channel, denoted by the shaded circle in Fig. 2, is calculated in the so-called chiral unitary approach [38-41]. The details of the formulation and calculation of the $N \bar{N} \rightarrow \Omega \bar{\Omega}$ scattering amplitude are given in Appendix D.

We fix the cutoff $t_{c}=(1.2 \mathrm{GeV})^{2}$, which is the upper boundary of the fit range of our $\pi \pi-K \bar{K}-\eta \eta$ scattering amplitude in the chiral unitary approach to the experimental $\pi \pi(J=0, I=$ 0 ) phase shift (Appendix F). The resulting $\mathcal{V}_{\mathrm{S}, 2 \mathrm{M}}$ in the region $t<0$ are shown in Fig. 3.

\section{E. Contact term}

The contact term $V_{\mathrm{C}}$ is straightforwardly constructed as

$$
\begin{aligned}
V_{\mathrm{C}}= & -c F(q)^{2} \bar{u}_{N}\left(\boldsymbol{p}^{\prime}, \lambda_{N}^{\prime}\right) u_{N}\left(\boldsymbol{p}, \lambda_{N}\right) \\
& \times \bar{u}_{\Omega \mu}\left(-\boldsymbol{p}^{\prime}, \lambda_{\Omega}^{\prime}\right) u_{\Omega}^{\mu}\left(-\boldsymbol{p}, \lambda_{\Omega}\right) .
\end{aligned}
$$

We here introduced the form factor $F(q)$ as in the $\eta$ and correlated two-meson exchanges. The unknown coupling constant $c$ is to be determined by the lattice QCD data as described in Sec. III.



FIG. 3. Lorentz invariant amplitudes $\mathcal{V}_{\mathrm{S}}$ and $\mathcal{V}_{2 \mathrm{M}}$ calculated with the dispersion relation as functions of the Mandelstam variable $t$. 
TABLE III. Channels for the $N \Omega$ coupled-channels scattering.

\begin{tabular}{ll}
\hline \hline 1 & $N \Omega\left({ }^{5} S_{2}\right)$ \\
2 & $\Lambda \Xi\left({ }^{3} D_{2}\right)$ \\
3 & $\Lambda \Xi\left({ }^{1} D_{2}\right)$ \\
4 & $\Sigma \Xi\left({ }^{3} D_{2}\right)$ \\
5 & $\Sigma \Xi\left({ }^{1} D_{2}\right)$ \\
6 & $\Lambda \Xi^{*}\left({ }^{5} S_{2}\right)$ \\
\hline \hline
\end{tabular}

\section{F. Projection to partial waves and coupling to inelastic channels}

The interaction terms above are constructed in terms of the helicity eigenstates in momentum space as

$$
V=V\left(\boldsymbol{p}^{\prime}, \lambda_{3}, \lambda_{4}, \boldsymbol{p}, \lambda_{1}, \lambda_{2}\right),
$$

where $\boldsymbol{p}$ and $\boldsymbol{p}^{\prime}$ are the relative momenta in the center-ofmass frame in the initial and final states, respectively, and $\lambda_{a}$ is the helicity for the $a$ th baryon in the baryon-baryon scattering $B_{1} B_{2} \rightarrow B_{3} B_{4}$. Now these interaction terms are projected to partial waves according to the method in Appendix C. As a result, the expression of the interaction reduces to

$$
V=V_{\alpha}\left(p^{\prime}, p\right)
$$

with $p^{(\prime)}=\left|\boldsymbol{p}^{(\prime)}\right|$ and $\alpha=\left(J, P, L^{\prime}, S^{\prime}, L, S\right)$, where $J, P$, $L^{(\prime)}$, and $S^{(\prime)}$ are total angular momentum, parity, spin, and orbital angular momentum of the two-body system in the initial (final) state, respectively.

In this study we focus on the $N \Omega$ interaction in its $S$ wave with $J^{P}=2^{+}\left({ }^{5} S_{2}\right.$ channel) where the attractive interaction is reported by the HAL QCD collaboration. Let us discuss contributions from the inelastic channels $\Lambda \Xi, \Sigma \Xi$, and $\Lambda \Xi^{*}$. Here we consider these channels with minimal orbital angular momenta; namely, $D, D$, and $S$ waves for the $\Lambda \Xi, \Sigma \Xi$, and $\Lambda \Xi^{*}$ channels, respectively. We note that, for $J^{P}=2^{+}$, there are two $D$-wave states with different spins $\left({ }^{3} D_{2}\right.$ and $\left.{ }^{1} D_{2}\right)$ in each of the $\Lambda \Xi$ and $\Sigma \Xi$ channels. In summary, we take into account the channels listed in Table III.

The evaluation of the inelastic contributions proceeds as follows: We first calculate a coupled-channel partial-wave projected interaction of the process $j(p) \rightarrow 1\left(p^{\prime}\right), V_{1 j}\left(p^{\prime}, p\right)$ where the channel index $j$ runs from 1 to 6 as listed in Table III. Owing to the time-reversal invariance of the strong interaction, we have a relation $V_{1 j}\left(p^{\prime}, p\right)=V_{j 1}\left(p, p^{\prime}\right)$. The transition to the inelastic channels is driven by the $K$ exchange as described in Sec. II G. We then evaluate the box diagram in Sec. II H to obtain the effective single-channel $N \Omega$ interaction in channel $1{ }^{1}$

\section{G. $K$ exchange for transition interaction}

The transition to inelastic channels with the $K$ exchange as shown in Fig. 1 (box) can be formulated in a similar manner to the case of the $\eta$ exchange. Here, for simplicity we make an approximation that the time component of the momentum

\footnotetext{
${ }^{1}$ The $N \Omega\left({ }^{5} D_{2}\right)$ and $N \Omega\left({ }^{3} D_{2}\right)$ channels couple to this sector through the tensor force in the $\eta$ exchange. We estimate this effect in Sec. IV A and show that their contributions are small.
}

transfer is zero, $q^{0} \approx 0$, and hence $q^{\mu} \approx\left(0, \boldsymbol{p}-\boldsymbol{p}^{\prime}\right)$. This can be guaranteed by the mass degeneracy in the SU(6) spin-flavor symmetry. Then, the transition terms are constructed as

$$
\begin{aligned}
V_{N \Omega \rightarrow \Lambda \Xi}= & -\frac{(D+3 F) f_{P B D}\left(m_{N}+m_{\Lambda}\right)}{2 \sqrt{3} f_{K} m_{\pi}} \frac{F(q)^{2}}{q^{2}+m_{K}^{2}} \\
& \times \bar{u}_{\Lambda}\left(\boldsymbol{p}^{\prime}, \lambda_{\Lambda}^{\prime}\right) \gamma_{5} u_{N}\left(\boldsymbol{p}, \lambda_{N}\right) \\
& \times \bar{u}_{\Xi}\left(-\boldsymbol{p}^{\prime}, \lambda_{\Xi}^{\prime}\right) q_{\mu} u_{\Omega}^{\mu}\left(-\boldsymbol{p}, \lambda_{\Omega}\right) \\
V_{N \Omega \rightarrow \Sigma \Xi}= & -\frac{\sqrt{3}(D-F) f_{P B D}\left(m_{N}+m_{\Sigma}\right)}{2 f_{K} m_{\pi}} \frac{F(q)^{2}}{q^{2}+m_{K}^{2}} \\
& \times \bar{u}_{\Sigma}\left(\boldsymbol{p}^{\prime}, \lambda_{\Sigma}^{\prime}\right) \gamma_{5} u_{N}\left(\boldsymbol{p}, \lambda_{N}\right) \\
& \times \bar{u}_{\Xi}\left(-\boldsymbol{p}^{\prime}, \lambda_{\Xi}^{\prime}\right) q_{\mu} u_{\Omega}^{\mu}\left(-\boldsymbol{p}, \lambda_{\Omega}\right) \\
V_{N \Omega \rightarrow \Lambda \Xi^{*}}= & -\frac{(D+3 F) f_{P D D}\left(m_{N}+m_{\Lambda}\right)\left(m_{\Xi^{*}}+m_{\Omega}\right)}{6 f_{K} m_{\pi}} \\
& \times \frac{F(q)^{2}}{q^{2}+m_{K}^{2}} \bar{u}_{\Lambda}\left(\boldsymbol{p}^{\prime}, \lambda_{\Lambda}^{\prime}\right) \gamma_{5} u_{N}\left(\boldsymbol{p}, \lambda_{N}\right) \\
& \times \bar{u}_{\Xi^{*} \mu}\left(-\boldsymbol{p}^{\prime}, \lambda_{\Xi^{*}}^{\prime}\right) \gamma_{5} u_{\Omega}^{\mu}\left(-\boldsymbol{p}, \lambda_{\Omega}\right)
\end{aligned}
$$

with $q \equiv|\boldsymbol{q}|=\left|\boldsymbol{p}-\boldsymbol{p}^{\prime}\right|$. We adopt the same form factor $F(q)$ with the other diagrams. Performing the partial-wave projection in Appendix C, we obtain $V_{1 j}\left(p^{\prime}, p\right)$.

\section{H. Inelastic contributions in box diagrams}

As we explained in Sec. II A, we consider the transition of the $N \Omega$ to the inelastic channels by the $K$ exchange but neglect the transition between the inelastic channels such as $\Lambda \Xi \rightarrow \Lambda \Xi$. In this case, the inelastic channels contribute to the $N \Omega\left({ }^{5} S_{2}\right)$ interaction only through the box diagrams in Fig. 1 (box). We can express this by using the partial-wave projected interaction in the previous section:

$$
V_{\mathrm{box}(j)}\left(E ; p^{\prime}, p\right)=\int_{0}^{\infty} \frac{d p^{\prime \prime}}{2 \pi^{2}} p^{\prime \prime 2} \frac{V_{1 j}\left(p^{\prime}, p^{\prime \prime}\right) V_{j 1}\left(p^{\prime \prime}, p\right)}{E-\mathcal{E}_{j}\left(p^{\prime \prime}\right)+i 0},
$$

with $j=2-6$. The on-shell energy $\mathcal{E}_{j}$ is

$$
\mathcal{E}_{j}(p) \equiv \sqrt{p^{2}+m_{j}^{2}}+\sqrt{p^{2}+m_{j}^{\prime 2}},
$$

with $m_{j}$ and $m_{j}^{\prime}$ being masses of particles in channel $j$ : $\left(m_{2}, m_{2}^{\prime}\right)=\left(m_{\Lambda}, m_{\Xi}\right),\left(m_{6}, m_{6}^{\prime}\right)=\left(m_{\Lambda}, m_{\Xi^{*}}\right)$, etc. We note that the interaction $V_{\operatorname{box}(j)}\left(E ; p^{\prime}, p\right)$ depends on the center-ofmass energy $E$. For a real energy $E>m_{i}+m_{i}^{\prime}$, the interaction $V_{\text {box }(j)}\left(E ; p^{\prime}, p\right)$ becomes complex according to the infinitesimal quantity $+i 0$ in the denominator. The imaginary part of the box interaction $V_{\text {box }(j)}$ represents the absorption of the $N \Omega$ system into the channel $j$.

\section{SCATTERING AMPLITUDE AND PARAMETER FIXING}

The $N \Omega\left({ }^{5} S_{2}\right)$ interaction we have formulated is composed of

$$
\begin{aligned}
V\left(E ; p^{\prime}, p\right)= & V_{\mathrm{A}}\left(p^{\prime}, p\right)+V_{\mathrm{B}}\left(p^{\prime}, p\right)+V_{\mathrm{C}}\left(p^{\prime}, p\right) \\
& +\sum_{j=2}^{6} V_{\mathrm{box}(j)}\left(E ; p^{\prime}, p\right) .
\end{aligned}
$$


Here $V_{\mathrm{A}}, V_{\mathrm{B}}, V_{\mathrm{C}}$ denote the contributions from the $\eta$ exchange, correlated two-meson exchange, and contact terms projected to the ${ }^{5} S_{2}$ channel, respectively. The box contribution $V_{\mathrm{box}(j)}$ was defined in Eq. (29).

One of the most important quantities calculated with this interaction is the $T$ matrix of the $N \Omega\left({ }^{5} S_{2}\right)$ scattering. In the present formulation, the $T$ matrix of the $N \Omega\left({ }^{5} S_{2}\right)$ scattering is a solution of the Lippmann-Schwinger equation in a single channel as follows:

$$
\begin{aligned}
T\left(E ; p^{\prime}, p\right)= & V\left(E ; p^{\prime}, p\right)+\int_{0}^{\infty} \frac{d p^{\prime \prime}}{2 \pi^{2}} p^{\prime \prime 2} \\
& \times \frac{V\left(E ; p^{\prime}, p^{\prime \prime}\right) T\left(E ; p^{\prime \prime}, p\right)}{E-\mathcal{E}_{N \Omega}\left(p^{\prime \prime}\right)+i 0},
\end{aligned}
$$

where $\mathcal{E}_{N \Omega}$ is the on-shell energy for the $N \Omega$ system,

$$
\mathcal{E}_{N \Omega}(p) \equiv \sqrt{p^{2}+m_{N}^{2}}+\sqrt{p^{2}+m_{\Omega}^{2}}
$$

The energy $E$ in Eq. (32) can be analytically continued to the complex plane. When we calculate the on-shell $T$ matrix $T_{\text {on }}$ for the $N \Omega\left({ }^{5} S_{2}\right)$ scattering above the threshold, the infinitesimal quantity $+i 0$ in the denominator specifies the boundary condition and gives the imaginary part of the $T$ matrix, which results in

$$
T_{\text {on }}(k)=\frac{K_{\text {on }}(k)}{1+K_{\text {on }}(k) \times i \rho(k) / 2},
$$

with the relative momentum $k$, on-shell $K$ matrix $K_{\text {on }}$, and phase space $\rho(k)$. The $K$ matrix is calculated with the integral equation

$$
\begin{aligned}
K\left(E ; p^{\prime}, p\right)= & V\left(E ; p^{\prime}, p\right)+\mathcal{P} \int_{0}^{\infty} \frac{d p^{\prime \prime}}{2 \pi^{2}} p^{\prime \prime 2} \\
& \times \frac{V\left(E ; p^{\prime}, p^{\prime \prime}\right) K\left(E ; p^{\prime \prime}, p\right)}{E-\mathcal{E}_{N \Omega}\left(p^{\prime \prime}\right)}
\end{aligned}
$$

where $\mathcal{P}$ stands for the principal value of the integral, and the on-shell part is

$$
K_{\text {on }}(k) \equiv K\left(\mathcal{E}_{N \Omega}(k) ; k, k\right) .
$$

The phase space $\rho(k)$ is defined as

$$
\rho(k) \equiv \frac{k}{\pi} \frac{\sqrt{\left(k^{2}+m_{N}^{2}\right)\left(k^{2}+m_{\Omega}^{2}\right)}}{\mathcal{E}_{N \Omega}(k)} .
$$

From the on-shell $T$ matrix, we can extract the threshold parameters for the $N \Omega$ scattering in nonrelativistic quantum mechanics such as the scattering length $a$ and effective range $r_{\text {eff }}$. In the present notation, the $N \Omega\left({ }^{5} S_{2}\right)$ scattering amplitude in nonrelativistic quantum mechanics, $f_{S}$, is expressed as

$$
f_{S}(k)=-\frac{1}{2 \pi} \frac{\sqrt{\left(k^{2}+m_{N}^{2}\right)\left(k^{2}+m_{\Omega}^{2}\right)}}{\mathcal{E}_{N \Omega}(k)} T_{\mathrm{on}}(k) .
$$

We can expand the inverse of the scattering amplitude, $f_{S}(k)^{-1}$, with respect to the relative momentum $k$ as

$$
f_{S}(k)^{-1}=-\frac{1}{a}-i k+\frac{1}{2} r_{\mathrm{eff}} k^{2}+O\left(k^{4}\right),
$$

where the scattering length $a$ and effective range $r_{\text {eff }}$ enter as the coefficients of the zeroth- and second-order terms, respectively. Therefore, we can calculate them from the behavior of the scattering amplitude at the threshold:

$$
\begin{aligned}
a & =-f_{S}(k=0), \\
r_{\text {eff }} & =\left[\frac{d^{2} f_{S}^{-1}}{d k^{2}}\right]_{k=0} .
\end{aligned}
$$

We note that the scattering length and effective range are in general complex if the interaction $V$ has imaginary part as the absorption into open channels.

Next, we would like to fix the model parameters in our potential: cutoff $\Lambda$ and coupling constant for the contact term $c$. Among the two parameters, the cutoff $\Lambda$ can be fixed to be a typical hadron scale. In the present study we take the value $\Lambda=1.0 \mathrm{GeV}$. For the coupling constant $c$, on the other hand, we need information on the $N \Omega\left({ }^{5} S_{2}\right)$ interaction. We employ the recent HAL QCD result on the scattering length from lattice QCD simulations with the nearly physical quark masses [30], in which the $N \Omega\left({ }^{5} S_{2}\right)$ system is found to be bound with very small binding energy and the system is almost in the unitary limit. In Ref. [30], they reported that the scattering length is $7.4 \pm 1.6 \mathrm{fm}$ at the time range $t=11$ of the lattice simulations. ${ }^{2}$ We reproduce this value by using our model but with hadron masses adjusted to the lattice simulations.

For the hadron masses in the lattice QCD simulations, we adopt $m_{N}^{\text {lat }}=964 \mathrm{MeV}$ and $m_{\Omega}^{\text {lat }}=1712 \mathrm{MeV}$ taken from Ref. [20], $m_{\Lambda}^{\text {lat }}=1123 \mathrm{MeV}, m_{\Sigma}^{\text {lat }}=1204 \mathrm{MeV}$, and $m_{\Xi}^{\text {lat }}=$ $1332 \mathrm{MeV}$ from Ref. [19], and $m_{\Xi^{*}}^{\text {lat }}=1580 \mathrm{MeV}$ from Fig. 1 of Ref. [43]. They are used to calculate the on-shell energies $\mathcal{E}_{j}$, which enter in the denominators of the $T$ matrix and box interactions, while we assume that the interaction (31) remains unchanged. In addition, to simulate the contributions from the $\Lambda \Xi$ and $\Sigma \Xi$ channels in finite volume in our framework, we take the real part of the box interaction $V_{\operatorname{box}(j)}$. Then, the scattering length is calculated as in Eq. (40) together with Eqs. (34)-(38) but with masses being $m_{N}^{\text {lat }}$ and $m_{\Omega}^{\text {lat }}$ instead of $m_{N}$ and $m_{\Omega}$, respectively.

In this condition, we obtain the scattering length $a=$ $7.4 \mathrm{fm}$ with the coupling constant $c=-22.1 \mathrm{GeV}^{-2}$. In the following, we adopt this value of the coupling constant $c=$ $-22.1 \mathrm{GeV}^{-2}$ together with the cutoff $\Lambda=1.0 \mathrm{GeV}$. We note that, if we keep the hadron masses in the lattice simulations but turn on the imaginary part of the box interaction, we obtain the scattering length $a=4.1-3.1 i \mathrm{fm}$.

\section{PROPERTIES OF THE $N \Omega$ INTERACTION}

Now that we have fixed parameters in our model, we discuss the properties of the $N \Omega\left({ }^{5} S_{2}\right)$ interaction. In the following, we

\footnotetext{
${ }^{2}$ We would like to thank T. Iritani and HAL QCD collaboration for providing us with the numerical value of the scattering length [42]. The HAL QCD collaboration provides a real-valued scattering length because of $N \Omega\left({ }^{5} S_{2}\right)$ single-channel analysis. We also note that the scattering length in our notation [see Eq. (39)] has the opposite sign with respect to that in HAL QCD.
} 


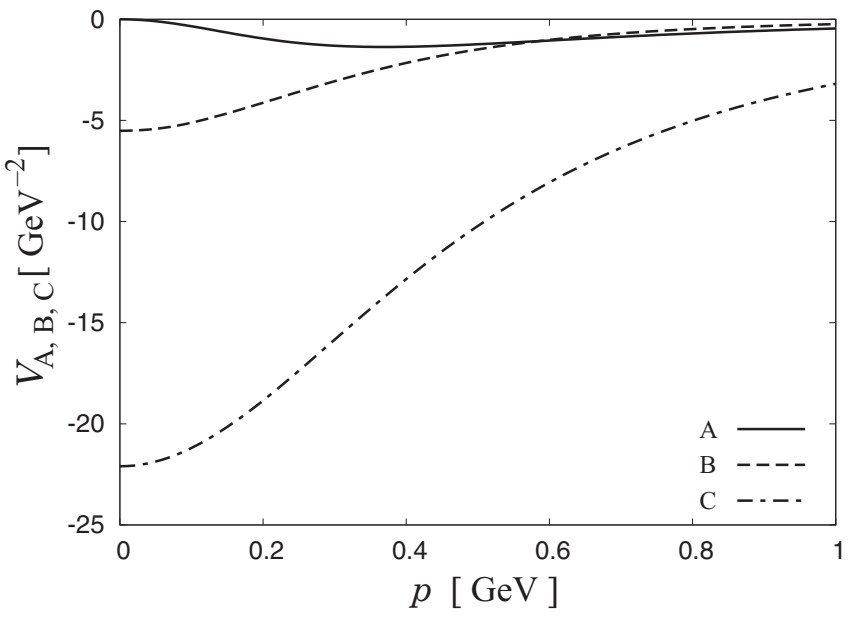

FIG. 4. The elastic contributions to the $N \Omega\left({ }^{5} S_{2}\right)$ interactions $V_{\mathrm{A}}(p, p), V_{\mathrm{B}}(p, p), V_{\mathrm{C}}(p, p)$ as functions of momentum $p$.

use the physical hadron masses in the isospin-symmetric limit summarized in Appendix A.

\section{A. Elastic contributions}

First we investigate properties of the $N \Omega\left({ }^{5} S_{2}\right)$ interaction in the elastic $N \Omega$ channel, i.e., the terms $V_{\mathrm{A}}\left(p^{\prime}, p\right), V_{\mathrm{B}}\left(p^{\prime}, p\right)$, $V_{\mathrm{C}}\left(p^{\prime}, p\right)$ in Eq. (31). We note that the terms $V_{\mathrm{A}}, V_{\mathrm{B}}, V_{\mathrm{C}}$ has no dependence on the energy $E$. The contributions $V_{\mathrm{A}}\left(p^{\prime}=p, p\right)$, $V_{\mathrm{B}}\left(p^{\prime}=p, p\right), V_{\mathrm{C}}\left(p^{\prime}=p, p\right)$ are plotted in Fig. 4 as functions of the momentum $p$. As one can see from the figure, the contact term $V_{\mathrm{C}}$ is strongly attractive and gives a dominant contribution. Other two terms, $V_{\mathrm{A}}$ and $V_{\mathrm{B}}$, give moderate attraction on top of the contact term. This finding of the weak $\eta$ and " $\sigma$ " exchanges is consistent with the calculation based on a quark model in Ref. [28]. The $\eta$ exchange interaction $V_{\mathrm{A}}$ is weak because the $\eta N N$ coupling constant is small. The correlated two-meson exchange interaction $V_{\mathrm{B}}$ is also weak. This is in contrast to the $N N$ case, in which the broad " $\sigma$ meson" plays an important role to generate $N N$ attraction in the intermediate range region. The weakness of the correlated two-meson exchange in the $N \Omega$ system is because the broad " $\sigma$ meson" cannot couple to the $\Omega$ via the $\pi \pi$ state [see Eq. (E11)]. Another resonance in the scalar-isoscalar channel, $f_{0}(980)$, has been considered to be a $K \bar{K}$ molecular state [44-46] and hence it can couple both to $N$ and $\Omega$. However, this contribution turns out to be also small, presumably because the heavier meson exchange acts only in the short-range (high-momentum) region.

To estimate the strength of the attraction, we calculate the volume integral of the interaction in the momentum space:

$$
\int_{0}^{\infty} d p V(p, p)
$$

The numerical results of the volume integrals of $V_{\mathrm{A}}, V_{\mathrm{B}}, V_{\mathrm{C}}$ are listed in the second, third, and fourth rows in Table IV, respectively. We can see that the contact term (C) is about ten times more attractive than the $\eta$ or correlated two-meson exchange. In other words, the lattice QCD scattering length [30] requires such attractive component represented by the
TABLE IV. Volume integral (42) of the $N \Omega$ interaction from each contribution in units of $\mathrm{GeV}^{-1}$. The energy is fixed as $E=$ $2550 \mathrm{MeV}, m_{N}+m_{\Omega}=2611.4 \mathrm{MeV}$, and $2650 \mathrm{MeV}$. Contributions from inelastic channels are evaluated as the box terms.

\begin{tabular}{lccc}
\hline \hline Contribution & $2550 \mathrm{MeV}$ & $m_{N}+m_{\Omega}$ & $2650 \mathrm{MeV}$ \\
\hline $\mathrm{A}$ & -1.11 & -1.11 & -1.11 \\
$\mathrm{~B}$ & -2.22 & -2.22 & -2.22 \\
$\mathrm{C}$ & -13.21 & -13.21 & -13.21 \\
$N \Omega\left({ }^{5} D_{2}\right)$ & -0.08 & -0.10 & $-0.12-0.01 i$ \\
$N \Omega\left({ }^{3} D_{2}\right)$ & -0.03 & -0.03 & $-0.04-0.00 i$ \\
$\Lambda \Xi\left({ }^{3} D_{2}\right)$ & $-1.41-0.55 i$ & $-1.32-0.94 i$ & $-1.19-1.13 i$ \\
$\Lambda \Xi\left({ }^{1} D_{2}\right)$ & $-0.92-0.37 i$ & $-0.86-0.62 i$ & $-0.78-0.75 i$ \\
$\Sigma \Xi\left({ }^{3} D_{2}\right)$ & $-0.24-0.02 i$ & $-0.28-0.09 i$ & $-0.28-0.15 i$ \\
$\Sigma \Xi\left({ }^{1} D_{2}\right)$ & $-0.16-0.01 i$ & $-0.18-0.06 i$ & $-0.18-0.10 i$ \\
$\Lambda \Xi{ }^{*}\left({ }^{5} S_{2}\right)$ & -0.53 & -0.67 & $-0.97-0.05 i$ \\
Total & $-19.89-0.95 i$ & $-19.97-1.72 i$ & $-20.08-2.18 i$ \\
\hline \hline
\end{tabular}

contact term, in addition to the conventional meson exchanges at long distance.

In addition to the $S$ wave, we examine the $D$-wave $N \Omega$ contribution as well because the $\eta$ exchange term $V_{\mathrm{A}}$ can mix the $S$ - and $D$-wave states owing to the tensor-force coupling, which is essential in the $N N$ system through the $\pi$ exchange. We note that there are two $D$-wave states with different spins in the $J^{P}=2^{+}$state, $N \Omega\left({ }^{5} D_{2}\right)$ and $N \Omega\left({ }^{3} D_{2}\right)$, to which we assign the channels $j=7$ and 8 , respectively. We calculate the $D$-wave contribution to the $S$ wave in the $N \Omega$ system through the box diagrams with the intermediate state being the $N \Omega\left({ }^{5} D_{2}\right)$ and $N \Omega\left({ }^{3} D_{2}\right)$ channels:

$$
V_{D}\left(E ; p^{\prime}, p\right)=\sum_{j=7}^{8} \int_{0}^{\infty} \frac{d p^{\prime \prime}}{2 \pi^{2}} p^{\prime \prime 2} \frac{V_{1 j}\left(p^{\prime}, p^{\prime \prime}\right) V_{j 1}\left(p^{\prime \prime}, p\right)}{E-\mathcal{E}_{N \Omega}\left(p^{\prime \prime}\right)+i 0}
$$

For the interaction $V_{j 1}(j=7$ and 8$)$ in the numerator of the integrand, we consider only the $N \Omega$ channel, $V_{\mathrm{A}}+V_{\mathrm{B}}+V_{\mathrm{C}}$, projected to the $S$ and $D$ waves in the initial and final states, respectively. We note that the effective interaction $V_{D}$ depends on the energy owing to the reduction of the $D$-wave channels.

The $D$-wave contribution to the $S$-wave interaction $V_{D}(E ; p, p)$ is plotted in Fig. 5 as a function of the momentum $p$. We fix the energy in the effective interaction as $E=$ $m_{N}+m_{\Omega}=2611.4 \mathrm{MeV}, 2550 \mathrm{MeV}$, and $2650 \mathrm{MeV}$. Note that the box term provides an imaginary part of the interaction above the threshold ( $E=2650 \mathrm{MeV})$. Comparing the result in Fig. 5 with those in Fig. 4 , we find that the $D$-wave contribution $\left(\sim-0.4 \mathrm{GeV}^{-2}\right.$ at $\left.p=0 \mathrm{GeV}\right)$ is very tiny with respect to the $S$-wave contact term $\left(\sim-22 \mathrm{GeV}^{-2}\right.$ at $\left.p=0 \mathrm{GeV}\right)$ and hence the $D$-wave contribution in the $S$-wave interaction is negligible. We can understand this behavior by the weak $\eta N N$ coupling compared with the $\pi N N$ coupling. We also find that the energy dependence of the $D$-wave contribution in the $S$-wave interaction is not significant.

To quantify the smallness of the $D$-wave contribution, we calculate the volume integral (42) of $V_{D}$ as listed in the fifth and sixth rows in Table IV. The volume integral from the $D$-wave contribution is only $\sim 1 \%$ of the contact-term contribution. 


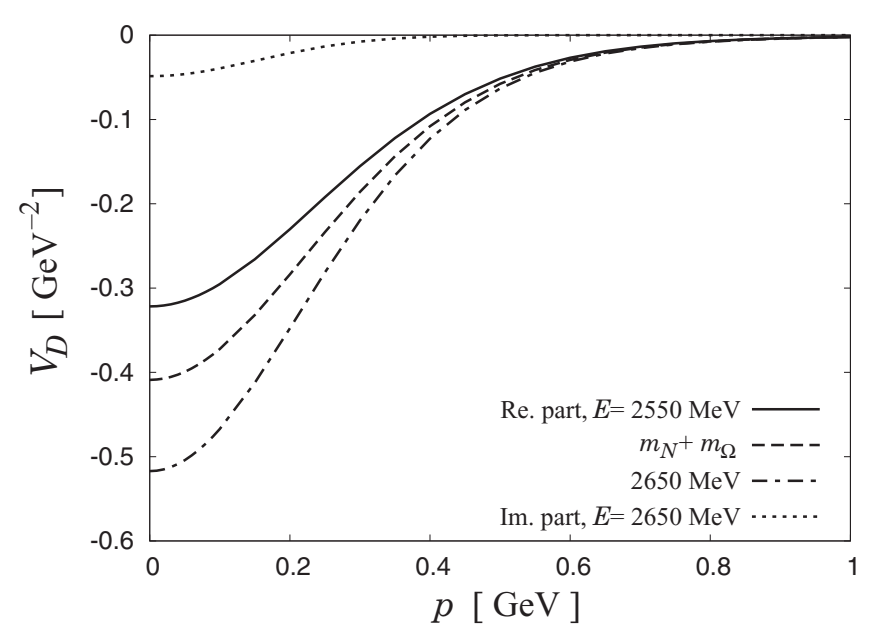

FIG. 5. The $D$-wave contribution to the $N \Omega\left({ }^{5} S_{2}\right)$ interaction $V_{D}(E ; p, p)$ as a function of momentum $p$. The energy in the effective interaction is fixed as $E=m_{N}+m_{\Omega}, 2550 \mathrm{MeV}$, and $2650 \mathrm{MeV}$. Because the energy is above the $N \Omega$ threshold, the contribution at $E=2650 \mathrm{MeV}$ has an imaginary part.

Based on these results, in the following discussions we neglect the $N \Omega\left({ }^{5} D_{2}\right)$ and $N \Omega\left({ }^{3} D_{2}\right)$ channels.

\section{B. Inelastic contributions}

Next we investigate the effects of the inelastic channels to the $N \Omega\left({ }^{5} S_{2}\right)$ interaction.

The contributions $V_{\mathrm{box}(j)}\left(E ; p^{\prime}=p, p\right)$ are plotted in Fig. 6 as functions of the momentum $p$. Here we show the sum of the ${ }^{3} D_{2}$ and ${ }^{1} D_{2}$ contributions in the $\Lambda \Xi$ and $\Sigma \Xi$ channels, for simplicity. The energy is fixed at threshold $E=m_{N}+m_{\Omega}$, thus the interaction which involves open $\Lambda \Xi$ or $\Sigma \Xi$ channel in the intermediate state has an imaginary part. From the real part of the interaction, we observe that the $\Lambda \Xi, \Sigma \Xi$, and $\Lambda \Xi^{*}$ channels assist the attraction of the

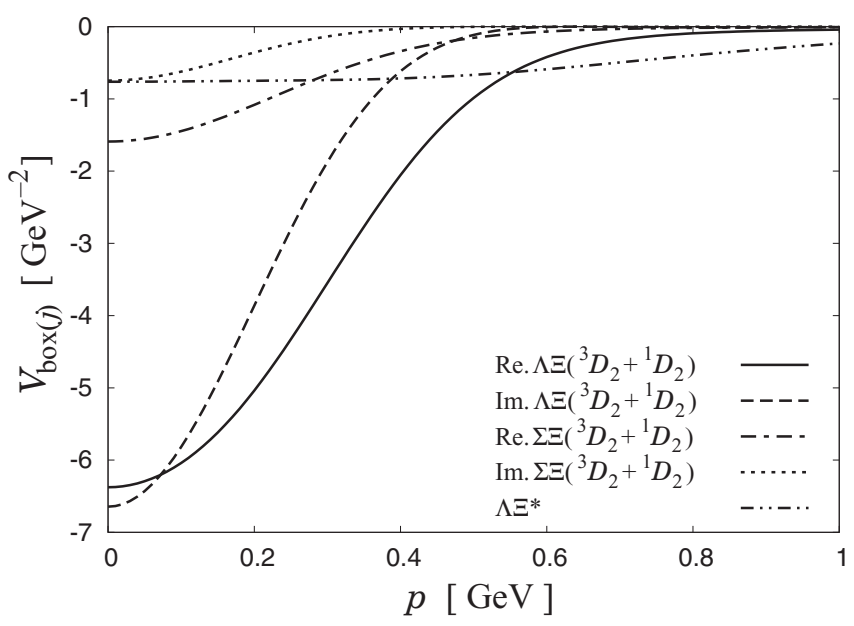

FIG. 6. The inelastic contributions to the $N \Omega\left({ }^{5} S_{2}\right)$ interaction $V_{\text {box }(j)}(E ; p, p)$ as functions of momentum $p$. The energy in the effective interaction is fixed as $E=m_{N}+m_{\Omega}$. In the figure $\Lambda \Xi$ and $\Sigma \Xi$ indicates the sum of ${ }^{3} D_{2}$ and ${ }^{1} D_{2}$ contributions, respectively.
$N \Omega\left({ }^{5} S_{2}\right)$ interaction. Among them, the $\Lambda \Xi$ channel gives the strongest attraction, which is comparable to the correlated two-meson exchange $V_{\mathrm{B}}$ (see Fig. 4). Even with the smaller energy denominator, the interaction of the intermediate $\Sigma \Xi$ channel is suppressed compared with the $\Lambda \Xi$ case by the smaller $K N \Sigma$ coupling: $(D+3 F) / 2 \sqrt{3} \approx 0.63$ for the $K N \Lambda$ coupling, and $\sqrt{3}(D-F) / 2 \approx 0.29$ for the $K N \Sigma$ coupling. The intermediate $\Lambda \Xi^{*}$ channel becomes significant only at higher momentum $p \gtrsim 0.6 \mathrm{GeV}$. As for the imaginary part of the interaction, the intermediate $\Lambda \Xi$ term gives larger contribution than the $\Sigma \Xi$ one, which indicates the $N \Omega\left({ }^{5} S_{2}\right)$ system mainly decays to the $\Lambda \Xi$ channel.

We calculate the volume integral (42) from the inelastic contributions, and the results are listed from the seventh to eleventh rows in Table IV. We can see that the $\Lambda \Xi$ channel gives the strongest attraction and absorption among the inelastic channels. The sum of the real parts of the volume integrals from the $\Lambda \Xi\left({ }^{3} D_{2}\right)$ and $\Lambda \Xi\left({ }^{1} D_{2}\right)$ contributions is similar magnitude to the volume integral from the correlated two-meson exchange $\left(-2.22 \mathrm{GeV}^{-1}\right)$. The imaginary part grows as the energy $E$ increases because a larger phase space can be utilized for a higher energy $E$. We can also understand from Table IV that the energy dependence of the box interaction is not significant. Indeed, when we vary the energy from $E=m_{N}+m_{\Omega}$ to 2550 or $2650 \mathrm{MeV}$, the shift of the volume integral in each contribution is only $\lesssim 1 \%$ of the total amount of the volume integral listed in the last row of Table IV.

\section{On-shell $N \Omega\left({ }^{5} S_{2}\right)$ scattering amplitude}

We then calculate the on-shell $N \Omega\left({ }^{5} S_{2}\right)$ scattering amplitude above the $N \Omega$ threshold and extract the scattering length and effective range.

The $N \Omega$ scattering amplitude $f_{S}(k)$ as a function of the relative momentum $k$ is obtained by the formula (38). Because the inverse of the scattering amplitude $f_{S}(k)^{-1}$ is useful to extract the scattering length and effective range, we show the result of the inverse of the scattering amplitude $f_{S}(k)^{-1}$ in Fig. 7 (solid and dash-dotted lines). Because the $N \Omega$ interaction is complex reflecting the absorption into open channels, $\Lambda \Xi$ and $\Sigma \Xi$, $f_{S}(k)^{-1}$ is complex even at the threshold $k=0 \mathrm{GeV}$, which leads to a complex scattering length. The real part of $f_{S}(k)^{-1}$ is negative at the threshold, which implies the existence of an $N \Omega$ quasibound state below the threshold, and it increases almost quadratically. In the same energy region, the imaginary part of $f_{S}(k)^{-1}$ almost linearly decreases as a function of $k$ like $-i k$. Because the energy dependence of $f_{S}(k)^{-1}$ at low energy is dictated by $-i k+r_{\mathrm{eff}} k^{2} / 2$ as shown Eq. (39), Fig. 7 indicates the imaginary part of the effective range is small. By using the formulas (40) and (41), we can calculate the scattering length $a$ and effective range $r_{\text {eff }}$, respectively. In our model we obtain $a=5.3-4.3 i \mathrm{fm}$ and $r_{\text {eff }}=0.74+0.04 i \mathrm{fm}$. We find that the real part of the effective range roughly corresponds to the length scale of the $N \Omega$ interaction, and the imaginary part is small, as expected. The magnitude of the scattering length is evidently larger than the interaction range, indicating that the $N \Omega$ scattering is close to the unitary limit. With these threshold parameters, the effective range expansion (39) reproduces the 


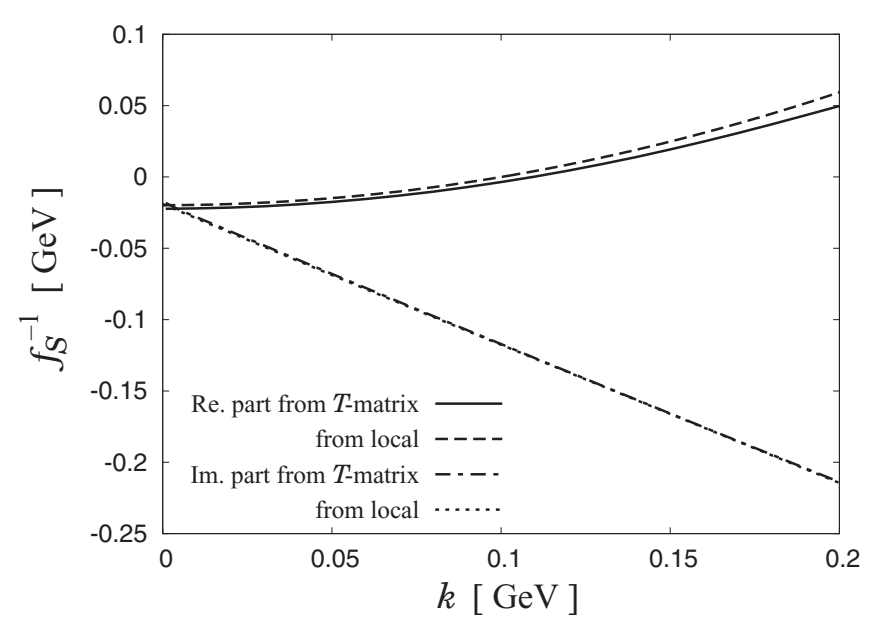

FIG. 7. Inverse of scattering amplitude $f_{S}^{-1}$. In addition to $f_{S}^{-1}$ calculated from the $T$ matrix, we also plot $f_{S}^{-1}$ from the equivalent local $N \Omega$ potential (see Sec. V). We note that the two lines of the imaginary parts almost overlap each other.

inverse of the scattering amplitude $f_{S}^{-1}$ fairly well in the energy range of Fig. 7.

\section{D. $N \Omega$ quasibound state}

Finally, by using the full $N \Omega\left({ }^{5} S_{2}\right)$ interaction (31) and solving the Lippmann-Schwinger equation (32), we search for a pole of the $N \Omega\left({ }^{5} S_{2}\right)$ quasibound state, indicated by the scattering length. As a result of the numerical calculation, we find a pole of the $N \Omega\left({ }^{5} S_{2}\right)$ quasibound state at $E_{\text {pole }}=$ $2611.3-0.7 i \mathrm{MeV}$ in the complex energy plane below the $N \Omega$ threshold $(2611.4 \mathrm{MeV})$. The pole exists in the first Riemann sheet of the $N \Omega$ channel and in the second Riemann sheets of the $\Lambda \Xi$ and $\Sigma \Xi$ channels. The pole position corresponds to the binding energy $0.1 \mathrm{MeV}$ and width $1.5 \mathrm{MeV}$.

In general, when one takes into account the imaginary part of the potential to represent absorption into open channels, the binding energy of a bound state in quantum mechanics decreases. In particular, a shallow bound state may disappear above the threshold. In the present case, the pole position of the $N \Omega\left({ }^{5} S_{2}\right)$ bound state would be $2611.0 \mathrm{MeV}$ if the imaginary part of the interaction coming from the box terms were absent. The imaginary part of the $N \Omega$ interaction reduces the binding energy of the $N \Omega$ bound state from 0.3 to $0.1 \mathrm{MeV}$. Therefore, we confirm that the absorptive effect by the $\Lambda \Xi$ and $\Sigma \Xi$ channels indeed acts repulsively, but the $N \Omega\left({ }^{5} S_{2}\right)$ quasibound state stays below the threshold.

The $N \Omega$ system is an isospin doublet, and there are two components, $p \Omega^{-}$and $n \Omega^{-}$. In addition to the strong interaction, in the $p \Omega^{-}$system, the attractive Coulomb interaction between $p$ and $\Omega^{-}$will assist the binding more. This point will be discussed at the end of this subsection with the wave function of the quasibound state.

To investigate the properties of the $N \Omega\left({ }^{5} S_{2}\right)$ quasibound state, we calculate its wave function from the residue of the $T$ matrix at the pole position, according to the approach in Ref. [47]. The off-shell $T$ matrix contains the pole in the following expression:

$$
T\left(E ; p^{\prime}, p\right)=\frac{\gamma\left(p^{\prime}\right) \gamma(p)}{E-E_{\text {pole }}}+\left(\text { regular at } E=E_{\text {pole }}\right) .
$$

The function $\gamma(p)$ is related to the radial part of the $N \Omega$ quasibound-state wave function in momentum space as

$$
R_{N \Omega}(p)=\frac{\sqrt{4 \pi} \gamma(p)}{E_{\text {pole }}-\mathcal{E}_{N \Omega}(p)} .
$$

An important point to be noted is that the wave function $R_{N \Omega}(p)$ is already normalized when extracted from the residue of the $T$ matrix, as the Lippmann-Schwinger equation (32) is inhomogeneous integral equation.

From the wave function $R_{N \Omega}(p)$ in momentum space we can calculate the wave function in coordinate space in a straightforward way:

$$
\begin{aligned}
\psi(r) & =\int \frac{d^{3} p}{(2 \pi)^{3}} e^{i \boldsymbol{p} \cdot \boldsymbol{r}} R_{N \Omega}(p) Y_{00} \\
& =\frac{Y_{00}}{2 \pi^{2} r} \int_{0}^{\infty} d p p \sin (p r) R_{N \Omega}(p),
\end{aligned}
$$

where $Y_{00} \equiv 1 / \sqrt{4 \pi}$ is the spherical harmonics. The norm of this wave function is expressed as

$$
X_{N \Omega}=\int d^{3} r[\psi(r)]^{2}=\int_{0}^{\infty} d r \mathrm{P}_{N \Omega}(r),
$$

where we define the "density distribution" $\mathrm{P}_{N \Omega}(r)$ :

$$
\mathrm{P}_{N \Omega}(r) \equiv 4 \pi r^{2}[\psi(r)]^{2} .
$$

We note the absence of the complex conjugate in Eqs. (44), (47), and (48), due to the unstable nature of the quasibound state. As a consequence, the wave functions $R_{N \Omega}(p), \psi(r)$, the density distribution $\mathrm{P}_{N \Omega}(r)$, and the norm $X_{N \Omega}$ are in general complex.

The norm $X_{N \Omega}$ from the $T$ matrix is called compositeness $[45,48,49]$ and quantitatively evaluates the importance of the $N \Omega$ degrees of freedom for the quasibound state in the employed model. The compositeness $X_{N \Omega}$ is unity for a purely $N \Omega$ state, but it deviates from unity when the interaction depends on the energy $E$ as a consequence of the effective reduction of the inelastic channels. In the present formulation, the quasibound state can have $\Lambda \Xi, \Sigma \Xi$, and $\Lambda \Xi^{*}$ components whose contributions are evaluated with $[50,51]$

$$
\begin{aligned}
X_{j}= & -\frac{1}{16 \pi^{5}} \int_{0}^{\infty} d p p^{2} R_{N \Omega}(p) \int_{0}^{\infty} d p^{\prime} p^{\prime 2} R_{N \Omega}\left(p^{\prime}\right) \\
& \times \frac{\partial V_{\mathrm{box}(j)}}{\partial E}\left(E_{\text {pole }} ; p^{\prime}, p\right)
\end{aligned}
$$

As a result, we obtain $X_{N \Omega}=1.00+0.00 i$ within three significant figures while we find that the others $X_{\Lambda \Xi}, X_{\Sigma \Xi}$, and $X_{\Lambda \Xi^{*}}$ are consistent with zero in this order. The result indicates that the quasibound state obtained in the present model is indeed composed of the $N \Omega$ channel.

Besides, using the weak-binding relation derived by Weinberg [1], the compositeness of a shallow bound state can be determined only by the observable quantities, the scattering length and the eigenenergy. The relation extended to the 


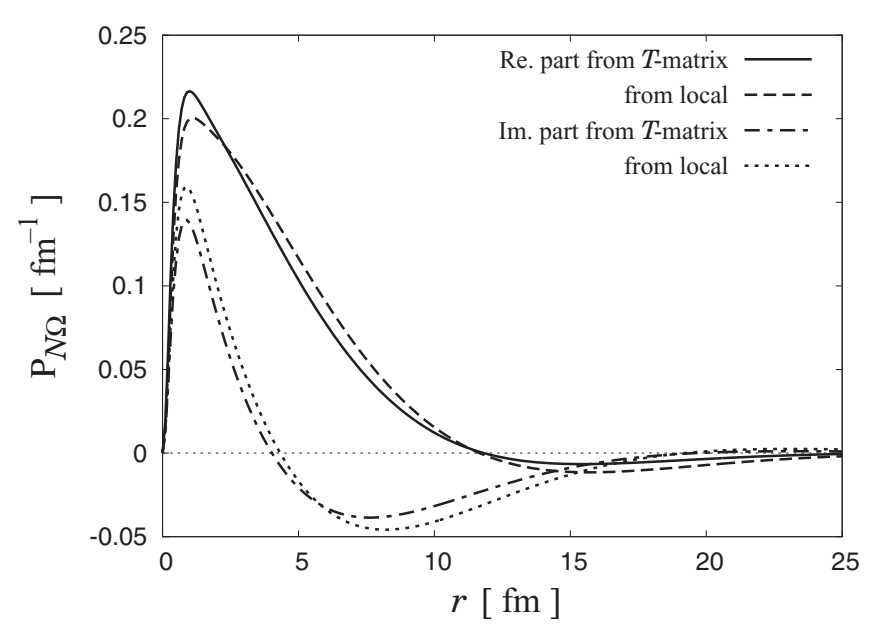

FIG. 8. Real and imaginary parts of the density distribution $\mathrm{P}_{N \Omega}$ for the $N \Omega\left({ }^{5} S_{2}\right)$ quasibound state as functions of radial coordinate $r$. In addition to $\mathrm{P}_{N \Omega}$ calculated from the $T$ matrix, we also plot $\mathrm{P}_{N \Omega}$ from the equivalent local $N \Omega$ potential (see Sec. V).

quasibound state with the finite decay width is given as [52,53]

$$
\begin{aligned}
& a=R\left[\frac{2 X_{N \Omega}^{\mathrm{wb}}}{1+X_{N \Omega}^{\mathrm{wb}}}+O\left(\left|\frac{R_{\mathrm{typ}}}{R}\right|\right)+O\left(\left|\frac{l}{R}\right|^{3}\right)\right], \\
& R \equiv 1 / \sqrt{-2 \mu E_{h}}, \quad l \equiv 1 / \sqrt{2 \mu \omega},
\end{aligned}
$$

where $X_{N \Omega}^{\mathrm{wb}}$ is the compositeness for the $N \Omega$ channel, $E_{h}=E_{\text {pole }}-m_{N}-m_{\Omega}=-0.1-0.7 i \mathrm{MeV} \quad$ is the eigenenergy of the bound state measured from the $N \Omega$ threshold energy, $\mu=m_{N} m_{\Omega} /\left(m_{N}+m_{\Omega}\right)$ is the reduced mass, $R_{\text {typ }}$ is the typical length scale of the interaction, and $\omega=37.7 \mathrm{MeV}$ denotes the difference between the threshold energy of the $N \Omega$ channel and that of the nearest channel, $\Lambda \Xi^{*}$. We estimate $R_{\text {typ }}$ with the $\eta$ meson exchange interaction as $R_{\text {typ }}=1 / m_{\eta} \sim 0.4 \mathrm{fm}$. We find that $\left|R_{\text {typ }} / R\right| \sim 0.1$ and $|l / R|^{3} \sim 0.0$ are much smaller than unity, which justifies neglecting the second and third terms in Eq. (50) to calculate the compositeness of the $N \Omega$ quasibound state. Neglecting these correction terms and using the value of the scattering length $a=5.3-4.3 i \mathrm{fm}$ derived in the previous section, we obtain $X_{N \Omega}^{\mathrm{wb}}=1.1+0.1 i$. With this complex $X_{N \Omega}^{\mathrm{wb}}$, the real-valued compositeness, which is interpreted as the probability $[52,53]$, is calculated as $\tilde{X}_{N \Omega}^{\mathrm{wb}}=1.0$. This result indicates the dominance of the $N \Omega$ composite component, in agreement with the above calculation using the wave function.

We then plot the density distribution $\mathrm{P}_{N \Omega}(r)$ in coordinate space (48) in Fig. 8 (solid and dash-dotted lines). Because a certain amount of density exists beyond $r=10 \mathrm{fm}$, the $N \Omega$ quasibound state is a spatially extended system owing to the tiny binding energy from the $N \Omega$ threshold. The average of the "distance" between $N \Omega$ is $\sqrt{\left\langle r^{2}\right\rangle}=3.8-3.1 i \mathrm{fm}$, where we define $\left\langle r^{2}\right\rangle$ as

$$
\left\langle r^{2}\right\rangle \equiv \int_{0}^{\infty} d r r^{2} \mathrm{P}_{N \Omega}(r)
$$

Although the "distance" is complex due to the resonance nature, its absolute value largely exceeds the typical size of baryons of $0.8 \mathrm{fm}$. Meanwhile, as explained in Ref. [51], the dumping of the wave function outside the potential range is related to the standard expectation value of the average distance:

$$
\left\langle r_{\text {dump }}^{2}\right\rangle \equiv \frac{\int_{0}^{\infty} d r r^{2}\left|\mathrm{P}_{N \Omega}(r)\right|}{\int_{0}^{\infty} d r\left|\mathrm{P}_{N \Omega}(r)\right|}
$$

The result is $\left(\left\langle r_{\text {dump }}^{2}\right\rangle\right)^{1 / 2}=6.5 \mathrm{fm}$, which indicates dumping of the wave function to a very large distance compared with the typical hadron scale owing to the tiny binding energy.

We also estimate the shift of the binding energy by the Coulomb interaction in the $p \Omega^{-}$quasibound state. We calculate the shift of the bound-state eigenenergy, $\Delta E_{\text {Coulomb }}$, in a perturbation:

$$
\Delta E_{\text {Coulomb }} \equiv \int_{0}^{\infty} d r\left(-\frac{\alpha}{r}\right) \mathrm{P}_{N \Omega}(r)
$$

where $\alpha \approx 1 / 137$ is the fine-structure constant. The result is $\Delta E_{\text {Coulomb }}=-0.9-0.4 i \mathrm{MeV}$. Therefore, we conclude that both the binding energy and decay width will respectively shift $\sim+1 \mathrm{MeV}$ by the Coulomb interaction for the $p \Omega^{-}$bound state.

\section{EQUIVALENT LOCAL $N \Omega$ POTENTIAL}

The existence of the $N \Omega$ quasibound state below the threshold implies possible $\Omega$ nuclei, generated by the attractive $N \Omega$ interaction. In addition, such possible $\Omega$ nuclei would shift eigenenergies of $\Omega^{-}$atoms, Coulombic bound states of $\Omega^{-}$and nuclei. To study the few-body system of $\Omega$ in nuclei, it is useful to have a local $N \Omega$ potential in the Schrödinger equation for which several established techniques to perform rigorous few-body calculations are available. On the other hand, because the momentum-space $N \Omega$ interaction in the present formulation (31) is a function not only of the momentum transfer $\left|\boldsymbol{p}-\boldsymbol{p}^{\prime}\right|$ but also of the momenta $p$ and $p^{\prime}$ individually, it is in general nonlocal. In addition, the scattering equation (32) is formulated with semirelativistic kinematics for baryons. Here we construct a local potential which equivalently reproduces the $N \Omega\left({ }^{5} S_{2}\right)$ scattering amplitude in this study. We first determine the local potential through the matching with the interaction (31), and then check whether the low-energy observables are properly reproduced.

We consider a local potential in the $S$-wave Schrödinger equation

$$
\left[-\frac{1}{2 \mu r} \frac{d^{2}}{d r^{2}} r+m_{N}+m_{\Omega}+V_{\text {local }}(r)\right] \psi(r)=E \psi(r),
$$

where $r$ is the relative coordinate of the $N \Omega$ system and the reduced mass is defined as $\mu=m_{N} m_{\Omega} /\left(m_{N}+m_{\Omega}\right)$. Note that the mass energy is included in the Hamiltonian in order to be consistent with the definition of $E$ in this paper. To parametrize $V_{\text {local }}(r)$, we introduce an analytic potential in momentum space as a superposition of nine Yukawa terms with different exchanged mass $m_{n}$ :

$$
\tilde{V}_{\text {local }}(q)=\sum_{n=1}^{9} \frac{C_{n}}{q^{2}+m_{n}^{2}}\left(\frac{\Lambda^{2}}{\Lambda^{2}+q^{2}}\right)^{2},
$$


where $q$ is the momentum transfer, $\Lambda$ is a cutoff, and $C_{n}$ are the strength parameters of the local potential. This local potential in coordinate space is expressed as

$$
V_{\text {local }}(r)=\int \frac{d^{3} q}{(2 \pi)^{3}} e^{-i \boldsymbol{q} \cdot \boldsymbol{r}} \tilde{V}_{\text {local }}(q)=\frac{1}{4 \pi r} \sum_{n=1}^{9} C_{n}\left(\frac{\Lambda^{2}}{\Lambda^{2}-m_{n}^{2}}\right)^{2}\left[e^{-m_{n} r}-\frac{\left(\Lambda^{2}-m_{n}^{2}\right) r+2 \Lambda}{2 \Lambda} e^{-\Lambda r}\right]
$$

To determine the strength parameters $C_{n}$, we project the momentum-space potential to the $S$ wave as

$$
\begin{aligned}
V_{\mathrm{NR}}\left(p^{\prime}, p\right)= & \frac{1}{2} \int_{-1}^{1} d \cos \theta \tilde{V}_{\mathrm{local}}\left(\sqrt{p^{2}-2 p p^{\prime} \cos \theta+p^{\prime 2}}\right) \\
= & \frac{1}{4 p p^{\prime}} \sum_{n=1}^{9} C_{n}\left(\frac{\Lambda^{2}}{\Lambda^{2}-m_{n}^{2}}\right)^{2}\left\{\ln \left[\frac{\left(p+p^{\prime}\right)^{2}+m_{n}^{2}}{\left(p-p^{\prime}\right)^{2}+m_{n}^{2}}\right]-\ln \left[\frac{\left(p+p^{\prime}\right)^{2}+\Lambda^{2}}{\left(p-p^{\prime}\right)^{2}+\Lambda^{2}}\right]\right. \\
& \left.+\frac{\Lambda^{2}-m_{n}^{2}}{\left(p+p^{\prime}\right)^{2}+\Lambda^{2}}-\frac{\Lambda^{2}-m_{n}^{2}}{\left(p-p^{\prime}\right)^{2}+\Lambda^{2}}\right\} .
\end{aligned}
$$

The $S$-wave Lippmann-Schwinger equation to obtain the $T$ matrix $T_{\mathrm{NR}}$, corresponding to the Schrödinger equation (55), is expressed as

$$
\begin{aligned}
T_{\mathrm{NR}}\left(E ; p^{\prime}, p\right)= & V_{\mathrm{NR}}\left(p^{\prime}, p\right)+\int_{0}^{\infty} \frac{d p^{\prime \prime}}{2 \pi^{2}} p^{\prime \prime 2} \\
& \times \frac{V_{\mathrm{NR}}\left(p^{\prime}, p^{\prime \prime}\right) T_{\mathrm{NR}}\left(E ; p^{\prime \prime}, p\right)}{E-\mathcal{E}_{\mathrm{NR}}\left(p^{\prime \prime}\right)+i 0},
\end{aligned}
$$

where the nonrelativistic on-shell energy is $\mathcal{E}_{\mathrm{NR}}(p) \equiv m_{N}+$ $m_{\Omega}+p^{2} /(2 \mu)$. We determine $C_{n}$ by the matching of $V_{\mathrm{NR}}\left(p^{\prime}, p\right)$ with $V\left(E ; p^{\prime}, p\right)$ in Eq. (31) at the threshold energy $E=m_{N}+m_{\Omega}$ as

$$
V\left(E=m_{N}+m_{\Omega} ; p^{\prime}, p\right)=f(p) f\left(p^{\prime}\right) V_{\mathrm{NR}}\left(p^{\prime}, p\right),
$$

with a factor to compensate the difference of the kinematics,

$$
f(p) \equiv \sqrt{\frac{\mathcal{E}_{N \Omega}(p)-m_{N}-m_{\Omega}}{p^{2} /(2 \mu)}} .
$$

With the factors $f(p) f\left(p^{\prime}\right)$, Eq. (59) coincides with the Lippmann-Schwinger equation (32) at the threshold.

We set the cutoff as the same value with $V\left(E ; p^{\prime}, p\right)$ in Eq. (31), $\Lambda=1 \mathrm{GeV}$, and the mass parameters are chosen to be $m_{n}=n \times(100 \mathrm{MeV})$ to cover the relevant ranges of the $N \Omega$ interaction. Then, we fit the coefficients $C_{n}$ to satisfy the condition (60). With nine terms in Eq. (56), we can reproduce each component of $N \Omega\left({ }^{5} S_{2}\right)$ interaction in Eq. (31) fairly well in the whole $p$ - $p^{\prime}$ plane. As a result of the best fit, we obtain the parameters $C_{n}$ listed in Table V.

Now we check that the local potential $V_{\text {local }}(r)$ well reproduces properties of the $N \Omega\left({ }^{5} S_{2}\right)$ scattering amplitude around the threshold energy. Because we neglect the energy dependence of the potential, the local potential cannot be extrapolated to the energy region far away from the threshold. In the following, we examine the eigenenergy of the $N \Omega$ quasibound state and the low-energy scattering with momentum $k \leqslant 0.2 \mathrm{GeV}$.

First, we solve the Schrödinger equation (55) with the local potential and obtain a quasibound state with eigenenergy $E=2611.4-0.7 i \mathrm{MeV}$, which reproduces the pole position of the $T$ matrix in Eq. (32) to an accuracy of $0.1 \mathrm{MeV}$. From the wave function of the quasibound state, we calculate the density distribution $\mathrm{P}_{N \Omega}$ as in Eq. (48) and normalize it by the condition $X_{N \Omega}=1$ in Eq. (47). The real and imaginary parts of the resulting density distribution are plotted in Fig. 8 by the dashed and dotted lines, respectively. We can see that the density distribution from the local potential is very similar to that from the $T$ matrix. We also calculate the "distance" between $N \Omega$, which results in $\sqrt{\left\langle r^{2}\right\rangle}=2.8-4.5 i \mathrm{fm}$ and $\left(\left\langle r_{\text {dump }}^{2}\right\rangle\right)^{1 / 2}=7.4 \mathrm{fm}$ in the prescriptions of Eqs. (52) and (53), respectively. These values are in fair agreement with those from the $T$ matrix as well.

Let us switch on the Coulomb potential $V_{\text {Coulomb }}(r)=-\alpha / r$ for the $p \Omega^{-}$system. In the calculation of the energy shift in a perturbation of Eq. (54), we would obtain a similar result as in the previous section, because of the similarity of the density distributions $\mathrm{P}_{N \Omega}$. Instead of such a perturbative calculation, we can easily perform the full calculation in the present case by solving the Schrödinger equation (55) with $V_{\text {Coulomb }}+$ $V_{\text {local }}$. As a result of the full calculation, the eigenenergy moves to $2610.5-1.0 i \mathrm{MeV}$, where the binding energy and decay width shift $+0.9 \mathrm{MeV}$ and $+0.6 \mathrm{MeV}$, respectively. The increase of the binding energy is a natural consequence of the attractive Coulomb interaction. The Coulomb attraction induces the shrinkage of the wave function of the $N \Omega$ system, which leads to the increase of the decay width due to the enlarged overlap of two particles. The result of the shift of the eigenenergy indicates that the perturbative calculation of Eq. (54) gives a good estimation.

Second, we calculate the $S$-wave scattering amplitude $f_{S}(k)$ from the asymptotic behavior of the wave function $\psi(r)$ at energy $E$ with the local potential $V_{\text {local }}(r)$. The resulting $f_{S}(k)^{-1}$ is plotted in Fig. 7 as the dashed and dotted lines. We find that $f_{S}(k)^{-1}$ nicely reproduces the result from the $T$ matrix, which means that the local potential $V_{\text {local }}$ is accurate enough to describe the $N \Omega\left({ }^{5} S_{2}\right)$ scattering near the threshold $k \leqslant 0.2 \mathrm{GeV}$. With $f_{S}(k)^{-1}$ from the local potential $V_{\text {local }}(r)$, we evaluate the scattering length and effective range as $a=5.2-5.0 i \mathrm{fm}$ and $r_{\text {eff }}=0.78+0.06 i \mathrm{fm}$, in fair agreement with those from the $T$ matrix. Note that the value of the scattering length is sensitive to the small modification of the system, reflecting the divergence in the unitary limit. 
TABLE V. Parameters $C_{n}$ for an equivalent local $N \Omega$ interaction. Other quantities in Eq. (56) are fixed as $\Lambda=1 \mathrm{GeV}$ and $m_{n}=n \times$ $(100 \mathrm{MeV})$.

\begin{tabular}{rrrrrrrr}
\hline \hline$n$ & \multicolumn{1}{c}{$\mathrm{A}$} & \multicolumn{1}{c}{$\mathrm{B}$} & $\mathrm{C}$ & box $\Lambda \Xi\left({ }^{3} D_{2}+{ }^{1} D_{2}\right)$ & box $\Sigma \Xi\left({ }^{3} D_{2}+{ }^{1} D_{2}\right)$ & box $\Lambda \Xi^{*}$ & \multicolumn{1}{c}{ Total } \\
\hline 1 & 0.02 & 0.06 & 0.07 & $-0.04+0.00 i$ & $-0.01+0.00 i$ & 0.04 & -4.30 \\
2 & -2.37 & -6.21 & -6.48 & $4.67-0.19 i$ & $0.93+0.05 i$ & $-13.76-0.14 i$ \\
3 & 57.03 & 160.19 & 131.39 & $-121.94+5.34 i$ & $-24.01-1.24 i$ & 104.15 & $306.81+4.10 i$ \\
4 & -556.75 & -1680.33 & -1021.60 & $1304.16-59.70 i$ & $251.48+12.83 i$ & -1026.45 & $-2729.49-46.87 i$ \\
5 & 2699.73 & 8765.95 & 3548.93 & $-6980.93+287.70 i$ & $-1313.42-62.87 i$ & 5024.22 & $11744.48+224.83 i$ \\
6 & -7052.95 & -24755.80 & -5159.25 & $20223.50-534.01 i$ & $3719.98+167.01 i$ & -13263.90 & $-26288.42-367.01 i$ \\
7 & 10055.50 & 38369.80 & 667.40 & $-31881.20+69.44 i$ & $-5772.02-262.54 i$ & 19118.60 & $30558.08-193.09 i$ \\
8 & -7304.99 & -30596.40 & 5175.64 & $25509.10+685.14 i$ & $4577.55+227.55 i$ & -14091.70 & $-16730.80+912.69 i$ \\
9 & 2096.47 & 9776.40 & -3446.89 & $-8069.25-460.13 i$ & $-1442.98-81.38 i$ & 4138.10 & $3051.85-541.51 i$ \\
\hline \hline
\end{tabular}

We show in Fig. 9 the equivalent local $N \Omega\left({ }^{5} S_{2}\right)$ potential in coordinate space $V_{\text {local }}(r)$ together with the contribution from the contact term $V_{\mathrm{C}}$. From the figure, we confirm that the strong attraction in the $N \Omega\left({ }^{5} S_{2}\right)$ interaction originates from the contact term $V_{\mathrm{C}}$ while other contributions give moderate attraction. The interaction range in Fig. 9 is consistent with the effective range $\sim 0.7 \mathrm{fm}$ obtained from the scattering amplitude $f_{S}$.

\section{CONCLUSION}

In this study we have investigated the $N \Omega\left({ }^{5} S_{2}\right)$ interaction based on a baryon-baryon interaction model with meson exchanges. The long-range part has been composed of the conventional mechanisms: exchanges of $\eta$ and " $\sigma$," i.e., correlated two mesons in the scalar-isoscalar channel. The shortrange part has been represented by the contact interaction. In addition, we have taken into account inelastic channels $\Lambda \Xi$, $\Sigma \Xi$, and $\Lambda \Xi(1530)$ which couple to the $N \Omega\left({ }^{5} S_{2}\right)$ system via $K$ exchange. The inclusion of the open channels, $\Lambda \Xi$ and $\Sigma \Xi$, is important to describe the absorption effects in the physical $N \Omega$ system. The unknown strength of the contact interaction was determined by fitting the scattering length of the HAL QCD result at the nearly physical quark masses.

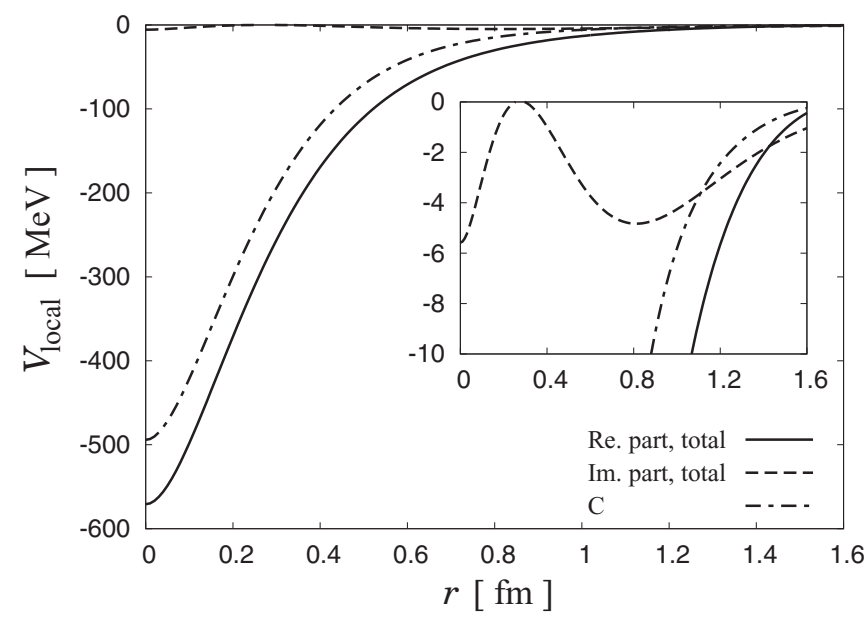

FIG. 9. Equivalent local $N \Omega$ potential in coordinate space $V_{\text {local }}(r)$. The contribution from the contact term $V_{\mathrm{C}}$ is also shown for comparison. The inset represents an enlarged figure.
The constructed $N \Omega\left({ }^{5} S_{2}\right)$ interaction was used to calculate the observable quantities at the physical point, including the absorption effects. For the $N \Omega\left({ }^{5} S_{2}\right)$ scattering, we have obtained the scattering length $a=5.3-4.3 i$ fm and the effective range $r_{\text {eff }}=0.74+0.04 i \mathrm{fm}$. The larger magnitude of the scattering length than the effective range indicates that the $N \Omega$ interaction is close to the unitary limit, and the positive real part indicates the existence of a shallow quasibound state below the threshold. Indeed, in searching for the pole of the scattering amplitude, we have found that the $N \Omega\left({ }^{5} S_{2}\right)$ quasibound state is generated with its eigenenergy $2611.3-0.7 i \mathrm{MeV}$, which corresponds to the binding energy $0.1 \mathrm{MeV}$ and the width $1.5 \mathrm{MeV}$. When the imaginary part of the interaction is switched off, we obtain a bound state at $2611.0 \mathrm{MeV}$. Thus, the imaginary part primarily induces the decay width, and slightly reduces the binding energy. The main decay mode is $\Lambda \Xi$, owing to the larger $K N \Lambda$ coupling than $K N \Sigma$ coupling. For the $p \Omega^{-}$bound state, the attractive Coulomb interaction further adds a shift of $\sim+1 \mathrm{MeV}$ both to the binding energy and decay width. The spatial size of the $N \Omega$ bound state will largely exceed the typical size of baryons.

We have discussed how the different mechanisms contribute to the $N \Omega$ interaction. It turns out that the attraction dominantly originates from the contact term. Other contributions, the $\eta$ exchange, correlated two-meson exchange, and box terms with inelastic channels in the intermediate states, give moderate attraction. Because we have considered all conventional mechanisms at the hadronic level, the discussion at the quark-gluon level would be necessary to clarify the origin of the $N \Omega$ attraction. Although the elimination of the inelastic channels induces the energy dependence of the single-channel $N \Omega$ interaction, the energy dependence has been found to be less than $1 \%$ in the energy region $50 \mathrm{MeV}$ above and below the threshold. We have found that the contribution from the $D$-wave $N \Omega$ states to the $N \Omega\left({ }^{5} S_{2}\right)$ interaction is negligible as well. These results justify constructing a single-channel $N \Omega$ potential in $S$ wave in the HAL QCD analysis [30,31].

We have constructed an equivalent local $N \Omega\left({ }^{5} S_{2}\right)$ potential, which will be useful to applications to few-body systems, such as possible $\Omega$ nuclei generated by the attractive $N \Omega$ interaction. To avoid the $S$-wave decays which would bring a large decay width, it is essential to align the spins of $\Omega$ and nucleons to the same direction so that the $N \Omega$ system couples to the $\Lambda \Xi$ and $\Sigma \Xi$ decay modes only in $D$ wave. In this sense, 
the $\Omega$-deuteron bound state with $J^{P}=5 / 2^{+}$will be the most plausible candidate of the $\Omega$ nuclei.

Finally, we remark on the possibility of the experimental investigation of the $N \Omega$ interaction and the quasibound state. Because the $N \Omega$ system has strangeness $S=-3$, practical candidate is the production in heavy-ion collisions [54,55]. Thanks to the small decay width, the $N \Omega$ quasibound state should be observed as a narrow peak in the invariant mass spectrum of the $\Lambda \Xi$ system near the $N \Omega$ threshold. In fact, the production yield of the $N \Omega$ bound state is estimated in Ref. [56] to be of the order of $10^{-3}$ per central collision at RHIC and LHC, assuming the binding energy of the $N \Omega$ system as $19 \mathrm{MeV}$. If the binding energy is much smaller, as we find in this paper, the production yield should be enhanced. Another tool, as discussed in Ref. [33], is the $p \Omega^{-}$two-body correlation which reflects the low-energy interaction of the $p \Omega^{-}$system. Because we have shown that the imaginary part of the scattering length has the same magnitude as the real part, the coupling to the open channels should be taken into account to study the realistic $p \Omega^{-}$correlation function.

\section{ACKNOWLEDGMENTS}

The authors acknowledge A. Ohnishi, F.-K. Guo, and E. Oset for fruitful discussions on the recent studies of the $N \Omega$ bound state, M. Oka on the dibaryons, and H. Kamano on the partial-wave analysis with helicity eigenstates. The authors are grateful to T. Doi, T. Iritani, and S. Gongyo for helpful discussions on the HAL QCD potential for the $N \Omega$ system. T.S. would like to thank the Yukawa Institute for Theoretical Physics, Kyoto University, where this work was initiated, for their kind hospitality during his visit. This work was partly supported by JSPS KAKENHI Grants No. JP15K17649, No. JP16K17694, and No. JP17J04333, by JSPS Japan-France Joint Research Project, and by the Yukawa International Program for Quark-Hadron Sciences (YIPQS).

\section{APPENDIX A: MASSES AND WIDTHS OF HADRONS}

In this study we use isospin symmetric masses for hadrons [34]: $m_{\pi}=138.0 \mathrm{MeV}, m_{K}=495.6 \mathrm{MeV}$, and $m_{\eta}=547.9 \mathrm{MeV}$ for mesons, $m_{N}=938.9 \mathrm{MeV}, m_{\Lambda}=$ 1115.7 MeV, $m_{\Sigma}=1193.2 \mathrm{MeV}$, and $m_{\Xi}=1318.3 \mathrm{MeV}$ for octet baryons, and $m_{\Delta}=1210.0 \mathrm{MeV}, m_{\Sigma^{*}}=1384.6 \mathrm{MeV}$, $m_{\Xi^{*}}=1533.4 \mathrm{MeV}$, and $m_{\Omega}=1672.5 \mathrm{MeV}$ for decuplet baryons. In addition, the widths of the decuplet baryons are $\Gamma_{\Delta}=100.0 \mathrm{MeV}, \Gamma_{\Sigma^{*}}=37.1 \mathrm{MeV}$, and $\Gamma_{\Xi^{*}}=9.5 \mathrm{MeV}$.

\section{APPENDIX B: CONVENTIONS}

In this Appendix we summarize our conventions of baryons used in this study.

Throughout this study the metric in four-dimensional Minkowski space is $g^{\mu \nu}=g_{\mu \nu}=\operatorname{diag}(1,-1,-1,-1)$, and the Einstein summation convention is used. The Dirac matrices $\gamma^{\mu}$ satisfy

$$
\left\{\gamma^{\mu}, \gamma^{\nu}\right\}=2 g^{\mu \nu}
$$

In the present study we choose the standard representation for the Dirac matrices:

$$
\gamma^{0}=\left(\begin{array}{cc}
\mathbf{1} & 0 \\
0 & -\mathbf{1}
\end{array}\right), \quad \boldsymbol{\gamma}=\left(\begin{array}{cc}
0 & \boldsymbol{\sigma} \\
-\boldsymbol{\sigma} & 0
\end{array}\right),
$$

with the Pauli matrices $\sigma$, and

$$
\gamma^{5} \equiv i \gamma^{0} \gamma^{1} \gamma^{2} \gamma^{3}=\left(\begin{array}{ll}
0 & \mathbf{1} \\
\mathbf{1} & 0
\end{array}\right) .
$$

The Dirac spinors for a positive-energy solution are expressed as $u(\boldsymbol{p}, s)$ with its three-momentum $\boldsymbol{p}$ and helicity $\lambda$, and its normalization is

$$
\bar{u}\left(\boldsymbol{p}, \lambda^{\prime}\right) u(\boldsymbol{p}, \lambda)=\delta_{\lambda^{\prime} \lambda},
$$

where $\bar{u} \equiv u^{\dagger} \gamma^{0}$. We employ the following explicit form of the Dirac spinors

$$
u(\boldsymbol{p}, \lambda)=\sqrt{\frac{E(p)+M}{2 M}}\left(\begin{array}{c}
\chi_{\lambda} \\
\frac{\boldsymbol{\sigma} \cdot \boldsymbol{p}}{E(p)+M} \chi_{\lambda}
\end{array}\right),
$$

where $M$ is the mass of the particle, $p \equiv|\boldsymbol{p}|$, and $E(p) \equiv$ $\left(p^{2}+M^{2}\right)^{1 / 2}$. The two-component spinor $\chi_{\lambda}$ is chosen to be helicity eigenstates

$$
\begin{aligned}
& \chi_{+1 / 2}=\left(\begin{array}{c}
e^{-i \phi / 2} \cos (\theta / 2) \\
e^{+i \phi / 2} \sin (\theta / 2)
\end{array}\right), \\
& \chi_{-1 / 2}=\left(\begin{array}{c}
-e^{-i \phi / 2} \sin (\theta / 2) \\
e^{+i \phi / 2} \cos (\theta / 2)
\end{array}\right),
\end{aligned}
$$

where $\theta$ and $\phi$ are the polar and azimuthal angles of the momentum $\boldsymbol{p}$, respectively. In fact, $\chi_{\lambda}$ satisfies

$$
\frac{1}{2} \frac{\boldsymbol{\sigma} \cdot \boldsymbol{p}}{p} \chi_{ \pm 1 / 2}= \pm \frac{1}{2} \chi_{ \pm 1 / 2}
$$

Owing to this relation, one can simplify the spinors in Eq. (B5) as

$$
u(\boldsymbol{p}, \lambda)=\left(\begin{array}{c}
\sqrt{\frac{E(p)+M}{2 M}} \chi_{\lambda} \\
\sqrt{\frac{E(p)-M}{2 M}} 2 \lambda \chi_{\lambda}
\end{array}\right) .
$$

The Dirac spinors for a negative-energy solution is then calculated as $v(\boldsymbol{p}, \lambda) \equiv i \gamma^{2} u^{*}(\boldsymbol{p}, \lambda)$, or explicitly

$$
v(\boldsymbol{p}, \lambda)=\left(\begin{array}{l}
-\sqrt{\frac{E(p)-M}{2 M}} \chi_{-\lambda} \\
\sqrt{\frac{E(p)+M}{2 M}} 2 \lambda \chi_{-\lambda}
\end{array}\right),
$$

which is normalized as

$$
\bar{v}\left(\boldsymbol{p}, \lambda^{\prime}\right) v(\boldsymbol{p}, \lambda)=-\delta_{\lambda^{\prime} \lambda} .
$$

The Dirac spinors satisfy the following relations:

$$
(\not p-M) u(\boldsymbol{p}, \lambda)=0, \quad(\not p+M) v(\boldsymbol{p}, \lambda)=0,
$$

and

$$
\begin{aligned}
& \sum_{\lambda} u(\boldsymbol{p}, \lambda) \bar{u}(\boldsymbol{p}, \lambda)=\frac{\not p+M}{2 M}, \\
& \sum_{\lambda} v(\boldsymbol{p}, \lambda) \bar{v}(\boldsymbol{p}, \lambda)=\frac{\not p-M}{2 M},
\end{aligned}
$$


with $p^{\mu}=(E(p), \boldsymbol{p})$ and $\not p \equiv p_{\mu} \gamma^{\mu}$.

Next, $e^{\mu}(\boldsymbol{p}, \lambda)$ denotes the polarization vectors for the spin 1 particle of three-momentum $\boldsymbol{p}$, helicity $\lambda$, and mass $M$. The vectors are chosen to be helicity eigenstates:

$$
\begin{aligned}
e^{\mu}(\boldsymbol{p}, \pm 1)= & \frac{ \pm 1}{\sqrt{2}}(0,-\cos \phi \cos \theta \pm i \sin \phi, \\
& -\sin \phi \cos \theta \mp i \cos \phi, \sin \theta), \\
e^{\mu}(\boldsymbol{p}, 0)= & \frac{E(p)}{M}\left(\frac{p}{E(p)}, \cos \phi \sin \theta, \sin \phi \sin \theta, \cos \theta\right) .
\end{aligned}
$$

These are obtained by boosting the helicity eigenstates at the rest frame of the particle $e^{\mu}(\mathbf{0}, \lambda)=\left(0, \hat{e}_{\lambda}\right)$ with the threevector $\hat{e}_{\lambda}$ :

$$
\begin{aligned}
& \hat{e}_{+1}=\left(-\frac{1}{\sqrt{2}},-\frac{i}{\sqrt{2}}, 0\right), \quad \hat{e}_{0}=(0,0,1), \\
& \hat{e}_{-1}=\left(\frac{1}{\sqrt{2}},-\frac{i}{\sqrt{2}}, 0\right),
\end{aligned}
$$

to the direction of the $z$ axis to have a momentum $p$ and then rotate to the direction of $(\theta, \phi)$, where the momentum is $\boldsymbol{p}=$ ( $p \cos \phi \sin \theta, p \sin \phi \sin \theta, p \cos \theta$ ). The polarization vectors are normalized as

$$
e^{\mu}\left(\boldsymbol{p}, \lambda^{\prime}\right) e_{\mu}^{*}(\boldsymbol{p}, \lambda)=-\delta_{\lambda^{\prime} \lambda},
$$

and satisfy the following relations:

$$
\begin{aligned}
p^{\mu} e_{\mu}(\boldsymbol{p}, \lambda) & =0, \\
\sum_{\lambda} e^{\mu}(\boldsymbol{p}, \lambda) e^{* \nu}(\boldsymbol{p}, \lambda) & =-g^{\mu \nu}+\frac{p^{\mu} p^{\nu}}{M^{2}} .
\end{aligned}
$$

Finally, the Rarita-Schwinger spinors for the spin- $\frac{3}{2}$ particle, $u^{\mu}(\boldsymbol{p}, \lambda)$, are constructed from the Dirac spinors $u(\boldsymbol{p}, \lambda)$ in Eq. (B8) and polarization vectors $e^{\mu}(\boldsymbol{p}, \lambda)$ in Eq. (B13) as

$$
u^{\mu}(\boldsymbol{p}, \lambda)=\sum_{\lambda_{1}, \lambda_{2}}\left\langle 11 / 2 \lambda_{1} \lambda_{2} \mid 3 / 2 \lambda\right\rangle e^{\mu}\left(\boldsymbol{p}, \lambda_{1}\right) u\left(\boldsymbol{p}, \lambda_{2}\right),
$$

with the Clebsch-Gordan coefficients $\left\langle j_{1} j_{2} m_{1} m_{2} \mid J M\right\rangle$. More explicitly, the Rarita-Schwinger spinors are

$$
\begin{aligned}
u^{\mu}(3 / 2) & =e^{\mu}(1) u(1 / 2), \\
u^{\mu}(1 / 2) & =\sqrt{\frac{2}{3}} e^{\mu}(0) u(1 / 2)+\sqrt{\frac{1}{3}} e^{\mu}(1) u(-1 / 2), \\
u^{\mu}(-1 / 2) & =\sqrt{\frac{1}{3}} e^{\mu}(-1) u(1 / 2)+\sqrt{\frac{2}{3}} e^{\mu}(0) u(-1 / 2), \\
u^{\mu}(-3 / 2) & =e^{\mu}(-1) u(-1 / 2),
\end{aligned}
$$

where we omitted the argument $\boldsymbol{p}$ for the Dirac spinors and polarization vectors. The Rarita-Schwinger spinors are normalized as

$$
\bar{u}^{\mu}\left(\boldsymbol{p}, \lambda^{\prime}\right) u_{\mu}(\boldsymbol{p}, \lambda)=-\delta_{\lambda^{\prime} \lambda},
$$

and satisfy the following relations:

$$
\begin{gathered}
(\not p-M) u_{\mu}(\boldsymbol{p}, \lambda)=0, \\
p^{\mu} u_{\mu}(\boldsymbol{p}, \lambda)=0, \quad \gamma^{\mu} u_{\mu}(\boldsymbol{p}, \lambda)=0 .
\end{gathered}
$$

In addition, in the rest frame of the particle, the RaritaSchwinger spinors satisfy $u^{0}(\mathbf{0}, \lambda)=0$ and

$$
\sum_{\lambda} u^{i}(\mathbf{0}, \lambda) \bar{u}^{j}(\mathbf{0}, \lambda)=\left(\begin{array}{cc}
2 \delta_{i j} / 3-i \epsilon_{i j k} \sigma^{k} / 3 & 0 \\
0 & 0
\end{array}\right) .
$$

In a similar manner, we can construct the Rarita-Schwinger spinors for the antiparticle $v^{\mu}(\boldsymbol{p}, \lambda)$ as

$$
v^{\mu}(\boldsymbol{p}, \lambda)=\sum_{\lambda_{1}, \lambda_{2}}\left\langle 11 / 2 \lambda_{1} \lambda_{2} \mid 3 / 2 \lambda\right\rangle e^{* \mu}\left(\boldsymbol{p}, \lambda_{1}\right) v\left(\boldsymbol{p}, \lambda_{2}\right),
$$

which satisfy the following normalization and relations:

$$
\begin{gathered}
\bar{v}^{\mu}\left(\boldsymbol{p}, \lambda^{\prime}\right) v_{\mu}(\boldsymbol{p}, \lambda)=\delta_{\lambda^{\prime} \lambda}, \\
(\not p+M) v_{\mu}(\boldsymbol{p}, \lambda)=0, \\
p^{\mu} v_{\mu}(\boldsymbol{p}, \lambda)=0, \quad \gamma^{\mu} v_{\mu}(\boldsymbol{p}, \lambda)=0 .
\end{gathered}
$$

\section{APPENDIX C: PARTIAL-WAVE PROJECTION OF INTERACTIONS}

In this Appendix we show formulas of the projection of baryon-baryon interactions to general partial waves. Here the baryon-baryon scatterings are denoted by $B_{1}\left(p_{1}^{\mu}, \lambda_{1}\right) B_{2}\left(p_{2}^{\mu}, \lambda_{2}\right) \rightarrow B_{3}\left(p_{3}^{\mu}, \lambda_{3}\right) B_{4}\left(p_{4}^{\mu}, \lambda_{4}\right)$, where the momenta $p_{a}^{\mu}\left(a=1,2,3\right.$, and 4) satisfy $p_{1}^{\mu}+p_{2}^{\mu}=p_{3}^{\mu}+p_{4}^{\mu}$ and $\lambda_{a}$ is the helicity of the baryon $B_{a}$. Since we consider scatterings in the center-of-mass frame, we can write the threemomenta as $\boldsymbol{p} \equiv \boldsymbol{p}_{1}=-\boldsymbol{p}_{2}$ and $\boldsymbol{p}^{\prime} \equiv \boldsymbol{p}_{3}=-\boldsymbol{p}_{4}$. Without loss of generality, we can choose the coordinates such that

$$
\boldsymbol{p}=(0,0, p), \quad \boldsymbol{p}^{\prime}=\left(p^{\prime} \sin \theta, 0, p^{\prime} \cos \theta\right),
$$

with the scattering angle $\theta$. The mass of the baryon $B_{a}$ is expressed as $m_{a}$.

We calculate the partial-wave matrix elements of the interaction $V_{\alpha}$ by following the Jacob-Wick formulation [57], where $\alpha$ specifies the quantum numbers of the system (see below). First, according to Feynman diagrams, we calculate the interactions in terms of the helicity eigenstates as $V\left(\boldsymbol{p}^{\prime}, \lambda_{3}, \lambda_{4}, \boldsymbol{p}, \lambda_{1}, \lambda_{2}\right)$, whose explicit forms are shown in the main part of this manuscript. Then, the interactions are projected to the total angular momentum $J$ as

$$
\begin{aligned}
& V^{J}\left(p^{\prime}, \lambda_{3}, \lambda_{4}, p, \lambda_{1}, \lambda_{2}\right) \\
& =\frac{\kappa\left(p^{\prime}, p\right)}{2} \int_{-1}^{1} d \cos \theta d_{\lambda_{1}-\lambda_{2} \lambda_{3}-\lambda_{4}}^{J}(\theta) \\
& \quad \times V\left(\boldsymbol{p}^{\prime}, \lambda_{3}, \lambda_{4}, \boldsymbol{p}, \lambda_{1}, \lambda_{2}\right),
\end{aligned}
$$

where $d_{m^{\prime} m}^{j}$ is the Wigner $d$ matrix and the factor $\kappa\left(p^{\prime}, p\right)$ is defined as

$$
\kappa\left(p^{\prime}, p\right) \equiv \sqrt{\frac{m_{1} m_{2} m_{3} m_{4}}{E_{1}(p) E_{2}(p) E_{3}\left(p^{\prime}\right) E_{4}\left(p^{\prime}\right)}},
$$


with $E_{a}(p) \equiv\left(p^{2}+m_{a}^{2}\right)^{1 / 2}$. The factor $\kappa$ was introduced so as to satisfy the optical theorem with the correct coefficients.

Finally, the interaction used for the Lippmann-Schwinger equation (32) is obtained as

$$
\begin{aligned}
V_{\alpha}\left(p^{\prime}, p\right)= & \sum_{\lambda_{1}, \lambda_{2}, \lambda_{3}, \lambda_{4}} \frac{\sqrt{(2 L+1)\left(2 L^{\prime}+1\right)}}{2 J+1} \\
& \times\left\langle j_{3} j_{4} \lambda_{3}-\lambda_{4} \mid S^{\prime} S_{z}^{\prime}\right\rangle\left\langle L^{\prime} S^{\prime} 0 S_{z}^{\prime} \mid J S_{z}^{\prime}\right\rangle \\
& \times\left\langle j_{1} j_{2} \lambda_{1}-\lambda_{2} \mid S S_{z}\right\rangle\left\langle L S 0 S_{z} \mid J S_{z}\right\rangle \\
& \times V^{J}\left(p^{\prime}, \lambda_{3}, \lambda_{4}, p, \lambda_{1}, \lambda_{2}\right)
\end{aligned}
$$

where $j_{a}$ is the spin of the baryon $B_{a}, L^{(\prime)}$, and $S^{(\prime)}$ are the orbital angular momentum and spin in the initial (final) state, respectively, $S_{z} \equiv \lambda_{1}-\lambda_{2}$, and $S_{z}^{\prime} \equiv \lambda_{3}-\lambda_{4}$.

Note that the orbital angular momentum $L$ and spin $S$ may take different values in the initial and final states as long as the total angular momentum $J$ and parity ${ }^{3} P=(-1)^{L}$ of the system are conserved. An important example is the mixing of the $S$ - and $D$-wave components for the $N \Omega$ system. In this sense, the quantum number is specified as $\alpha=\left(J, P, L^{\prime}, S^{\prime}, L, S\right)$.

\section{APPENDIX D: CORRELATED TWO-MESON EXCHANGE}

In this Appendix we summarize our formulation of the correlated two-meson exchange, for which we concentrate on the exchange of the scalar-isoscalar channel $\left(J^{P}, I\right)=\left(0^{+}, 0\right)$. The contribution of the correlated two-meson exchange was expressed as $\mathcal{V}_{\mathrm{S}, 2 \mathrm{M}}$ in Eq. (21). According to the dispersion relation (22), to calculate the correlated two-meson exchange taking place in the region $t<0$, we may consider the same amplitude but in $t>4 m_{\pi}^{2}$, which can be achieved in the $N \bar{N} \rightarrow \Omega \bar{\Omega}$ reaction as shown in Fig. 2 .

Let us formulate the $N \bar{N} \rightarrow \Omega \bar{\Omega}$ reaction in Fig. 2. We fix the nucleon momenta $p_{N}^{\mu}=(\sqrt{t} / 2, \boldsymbol{p})$ and $p_{\bar{N}}^{\mu}=(\sqrt{t} / 2,-\boldsymbol{p})$ with $\boldsymbol{p}=(0,0, p)$, and the $\Omega$ momenta $p_{\Omega}^{\mu}=\left(\sqrt{t} / 2, \boldsymbol{p}^{\prime}\right)$ and $p_{\bar{\Omega}}^{\mu}=\left(\sqrt{t} / 2,-\boldsymbol{p}^{\prime}\right)$ with $\boldsymbol{p}^{\prime}=\left(p^{\prime} \sin \theta, 0, p^{\prime} \cos \theta\right)$ and the scattering angle $\theta$. Because we concentrate on the scalar channel, the scattering amplitude of the $N \bar{N} \rightarrow \Omega \bar{\Omega}$ reaction can be evaluated as the matrix element of the corresponding $T$ matrix $\hat{T}^{J=0}$, which contains $\mathcal{V}_{\mathrm{S}, 2 \mathrm{M}}$ according to crossing symmetry:

$$
\begin{aligned}
& \left\langle\Omega \bar{\Omega}\left(\boldsymbol{p}^{\prime}, \lambda_{\Omega}, \lambda_{\bar{\Omega}}\right)\left|\hat{T}^{J=0}\right| N \bar{N}\left(\boldsymbol{p}, \lambda_{N}, \lambda_{\bar{N}}\right)\right\rangle \\
& =\delta_{\lambda_{N} \lambda_{\bar{N}}} \delta_{\lambda_{\Omega} \lambda_{\bar{\Omega}}} \times \bar{v}_{N}\left(-\boldsymbol{p}, \lambda_{N}\right) u_{N}\left(\boldsymbol{p}, \lambda_{N}\right) \\
& \quad \times\left[\mathcal{V}_{\mathrm{S}}(t) \bar{u}_{\Omega}^{\mu}\left(\boldsymbol{p}^{\prime}, \lambda_{\Omega}\right) v_{\Omega \mu}\left(-\boldsymbol{p}^{\prime}, \lambda_{\Omega}\right)\right. \\
& \left.\quad+\mathcal{V}_{2 \mathrm{M}}(t) \frac{P_{\mu} P_{v}}{m_{\Omega}^{2}} \bar{u}_{\Omega}^{\mu}\left(\boldsymbol{p}^{\prime}, \lambda_{\Omega}\right) v_{\Omega}^{\nu}\left(-\boldsymbol{p}^{\prime}, \lambda_{\Omega}\right)\right],
\end{aligned}
$$

where $P^{\mu} \equiv p_{N}^{\mu}+p_{\bar{N}}^{\mu}=(\sqrt{t}, \mathbf{0})$ and the constraints $\lambda_{N}=\lambda_{\bar{N}}$ and $\lambda_{\Omega}=\lambda_{\bar{\Omega}}$ are necessary to construct $J=0$. Here we note

\footnotetext{
${ }^{3}$ Note that all baryons in this study have positive parity.
}

some relations for the spinors:

$$
\begin{aligned}
\bar{v}_{N}(-\boldsymbol{p}, \pm 1 / 2) u_{N}(\boldsymbol{p}, \pm 1 / 2) & =\frac{i p}{m_{N}}, \\
\bar{u}_{\Omega}^{\mu}\left(\boldsymbol{p}^{\prime}, \pm 3 / 2\right) v_{\bar{\Omega} \mu}\left(-\boldsymbol{p}^{\prime}, \pm 3 / 2\right) & =\frac{i p^{\prime}}{m_{\Omega}}, \\
\bar{u}_{\Omega}^{\mu}\left(\boldsymbol{p}^{\prime}, \pm 1 / 2\right) v_{\bar{\Omega} \mu}\left(-\boldsymbol{p}^{\prime}, \pm 1 / 2\right) & =-\frac{i p^{\prime}}{3 m_{\Omega}}\left(1+\frac{4 p^{\prime 2}}{m_{\Omega}^{2}}\right),
\end{aligned}
$$

$$
\frac{P_{\mu} P_{v}}{m_{\Omega}^{2}} \bar{u}_{\Omega}^{\mu}\left(\boldsymbol{p}^{\prime}, \pm 3 / 2\right) v_{\Omega}^{v}\left(-\boldsymbol{p}^{\prime}, \pm 3 / 2\right)=0,
$$

$$
\frac{P_{\mu} P_{v}}{m_{\Omega}^{2}} \bar{u}_{\Omega}^{\mu}\left(\boldsymbol{p}^{\prime}, \pm 1 / 2\right) v_{\Omega}^{v}\left(-\boldsymbol{p}^{\prime}, \pm 1 / 2\right)=-\frac{2 i p^{\prime 3} t}{3 m_{\Omega}^{5}},
$$

where double-sign corresponds.

To calculate $\mathcal{V}_{\mathrm{S}, 2 \mathrm{M}}$ in the region $t<0$ via the dispersion relation (22), we need $\operatorname{Im} \mathcal{V}_{\mathrm{S}, 2 \mathrm{M}}(t)$ in $t>4 m_{\pi}^{2}$. For this purpose, we first recall the unitarity of the $S$ matrix: $\hat{S}^{\dagger} \hat{S}=1$. Expressing this relation in terms of the $T$ matrix of the $N \bar{N} \rightarrow \Omega \bar{\Omega}$ reaction in the scalar channel, we have

$$
\begin{aligned}
& i\left\langle\Omega \bar{\Omega}\left|\hat{T}^{J=0}\right| N \bar{N}\right\rangle-i\left\langle\Omega \bar{\Omega}\left|\hat{T}^{J=0 \dagger}\right| N \bar{N}\right\rangle \\
& \quad=\sum_{n} \rho_{n}(t) \theta\left(t-m_{\mathrm{th}(n)}^{2}\right)\left\langle\Omega \bar{\Omega}\left|\hat{T}^{J=0 \dagger}\right| n\right\rangle\left\langle n\left|\hat{T}^{J=0}\right| N \bar{N}\right\rangle,
\end{aligned}
$$

where we omitted the parameters $\left(\boldsymbol{p}^{\prime}, \lambda_{\Omega}, \lambda_{\bar{\Omega}}\right)$ for the $\Omega \bar{\Omega}$ state and $\left(\boldsymbol{p}, \lambda_{N}, \lambda_{\bar{N}}\right)$ for the $N \bar{N}$ state. On the right-hand side, $n=\pi \pi, K \bar{K}, \eta \eta, \ldots$ denote possible physical channels, $m_{\mathrm{th}(n)}$ is its threshold, and $\rho_{n}(t)$ is its phase space. In particular,

$$
\rho_{P \bar{P}}(t)=\frac{N_{P \bar{P}}}{8 \pi} \sqrt{\frac{t-4 m_{P}^{2}}{t}},
$$

for $n=P \bar{P}=\pi \pi, K \bar{K}, \eta \eta$, with the mass of the pseudoscalar meson $m_{P}$ and the symmetry factor for identical particles: $N_{\pi \pi}=N_{\eta \eta}=1 / 2$ and $N_{K \bar{K}}=1$. Therefore, by using the relation in Eq. (D1) and $\left\langle A\left|\hat{T}^{\dagger}\right| B\right\rangle=\langle B|\hat{T}| A\rangle^{*}$, we can rewrite (D7) as

$$
\begin{aligned}
& \delta_{\lambda_{N} \lambda_{\bar{N}}} \delta_{\lambda_{\Omega} \lambda_{\bar{\Omega}}} \times \bar{v}_{N}\left(-\boldsymbol{p}, \lambda_{N}\right) u_{N}\left(\boldsymbol{p}, \lambda_{N}\right) \\
& \times\left[-2 \operatorname{Im} \mathcal{V}_{\mathrm{S}}(t) \bar{u}_{\Omega}^{\mu}\left(\boldsymbol{p}^{\prime}, \lambda_{\Omega}\right) v_{\Omega \mu}\left(-\boldsymbol{p}^{\prime}, \lambda_{\Omega}\right)\right. \\
& \left.-2 \operatorname{Im} \mathcal{V}_{2 \mathrm{M}}(t) \frac{P_{\mu} P_{v}}{m_{\Omega}^{2}} \bar{u}_{\Omega}^{\mu}\left(\boldsymbol{p}^{\prime}, \lambda_{\Omega}\right) v_{\Omega}^{v}\left(-\boldsymbol{p}^{\prime}, \lambda_{\Omega}\right)\right] \\
= & \sum_{n} \rho_{n}(t) \theta\left(t-m_{\mathrm{th}(n)}^{2}\right)\left\langle n\left|\hat{T}^{J=0}\right| \Omega \bar{\Omega}\right\rangle^{*}\left\langle n\left|\hat{T}^{J=0}\right| N \bar{N}\right\rangle .
\end{aligned}
$$

This is a general formula to calculate $\operatorname{Im} \mathcal{V}_{\mathrm{S}, 2 \mathrm{M}}(t)$ in the region $t>4 m_{\pi}^{2}$. In the equation the magnitudes of the momenta of $N$ and $\Omega, p(t)$ and $p^{\prime}(t)$, respectively, take their on-shell values

$$
p(t)=\sqrt{\frac{t}{4}-m_{N}^{2}}, \quad p^{\prime}(t)=\sqrt{\frac{t}{4}-m_{\Omega}^{2}} .
$$

Note that the unitarity relation (D9) is defined above the kinematic threshold of the $N \bar{N} \rightarrow \Omega \bar{\Omega}$ reaction, i.e., $t>4 m_{\Omega}^{2}$. 
However, one can perform the analytic continuation into the pseudophysical region $t<4 m_{\Omega}^{2}$, where $p^{\prime}(t)$ [and $p(t)$ in $t<4 m_{N}^{2}$ ] is pure imaginary.

In general, $n$ in Eq. (D9) should run all possible physical channels, but it is well known that the scalar-isoscalar channel in the region $4 m_{\pi}^{2}<t \lesssim 1 \mathrm{GeV}^{2}$ is dominated by the contributions from the dynamics of $\pi \pi$ and coupled channels of two pseudoscalar mesons. Therefore, below we restrict the summation in Eq. (D9) to the physical $\pi \pi, K \bar{K}$, and $\eta \eta$ states.

Now our task is to calculate the scattering amplitudes of the $N \bar{N} \rightarrow P \bar{P}$ and $\Omega \bar{\Omega} \rightarrow P \bar{P}(P \bar{P}=\pi \pi, K \bar{K}, \eta \eta)$ reactions in the scalar-isoscalar channel. Here, to simplify the evaluation of the $N \Omega$ interaction from the $N \bar{N} \rightarrow \Omega \bar{\Omega}$ amplitude, $N \bar{N}$ is in the particle basis, i.e., $N \bar{N}=p \bar{p}$ or $n \bar{n}$, while the $P \bar{P}$ is in the isospin basis with $I=0$ :

$$
\begin{aligned}
|\pi \pi(\boldsymbol{k})\rangle= & -\frac{1}{\sqrt{3}} \mid \pi^{+}(\boldsymbol{k}) \pi^{-}(-\boldsymbol{k})+\pi^{0}(\boldsymbol{k}) \pi^{0}(-\boldsymbol{k}) \\
& \left.+\pi^{-}(\boldsymbol{k}) \pi^{+}(-\boldsymbol{k})\right\rangle, \\
|K \bar{K}(\boldsymbol{k})\rangle= & -\frac{1}{\sqrt{2}}\left|K^{+}(\boldsymbol{k}) K^{-}(-\boldsymbol{k})+K^{0}(\boldsymbol{k}) \bar{K}^{0}(-\boldsymbol{k})\right\rangle, \\
|\eta \eta(\boldsymbol{k})\rangle= & |\eta(\boldsymbol{k}) \eta(-\boldsymbol{k})\rangle,
\end{aligned}
$$

where $\boldsymbol{k}$ is the relative momentum of mesons. We explicitly write the off-shell amplitudes of the $N \bar{N} \rightarrow P \bar{P}$ and $\Omega \bar{\Omega} \rightarrow$ $P \bar{P}$ reactions as

$$
\begin{aligned}
& T_{N \bar{N} \rightarrow P \bar{P}}\left(t, k, p, \lambda_{N}\right)=\left\langle P \bar{P}(\boldsymbol{k})\left|\hat{T}^{J=0}\right| N \bar{N}\left(\boldsymbol{p}, \lambda_{N}, \lambda_{N}\right)\right\rangle, \\
& T_{\Omega \bar{\Omega} \rightarrow P \bar{P}}\left(t, k, p^{\prime}, \lambda_{\Omega}\right)=\left\langle P \bar{P}(\boldsymbol{k})\left|\hat{T}^{J=0}\right| \Omega \bar{\Omega}\left(\boldsymbol{p}^{\prime}, \lambda_{\Omega}, \lambda_{\Omega}\right)\right\rangle .
\end{aligned}
$$

Helicities are constrained as $\lambda_{N}=\lambda_{\bar{N}}$ and $\lambda_{\Omega}=\lambda_{\bar{\Omega}}$ so as to construct $J=0$. Because of this $S$-wave nature, the left-handside depends only on the magnitude of the momenta. Owing to the parity invariance of the underlying strong interaction, the amplitudes $T_{N \bar{N} \rightarrow P \bar{P}}$ and $T_{\Omega \bar{\Omega} \rightarrow P \bar{P}}$ have relations

$$
\begin{aligned}
& T_{N \bar{N} \rightarrow P \bar{P}}\left(t, k, p, \lambda_{N}\right)=T_{N \bar{N} \rightarrow P \bar{P}}\left(t, k, p,-\lambda_{N}\right), \\
& T_{\Omega \bar{\Omega} \rightarrow P \bar{P}}\left(t, k, p^{\prime}, \lambda_{\Omega}\right)=T_{\Omega \bar{\Omega} \rightarrow P \bar{P}}\left(t, k, p^{\prime},-\lambda_{\Omega}\right) .
\end{aligned}
$$

Therefore, while $T_{N \bar{N} \rightarrow P \bar{P}}$ does not depend on $\lambda_{N}, T_{\Omega \bar{\Omega} \rightarrow P \bar{P}}$ has two independent components of $\lambda_{\Omega}=3 / 2$ and $1 / 2$. Note that momenta $p, p^{\prime}$, and $k$ are independent of $t$ in the off-shell amplitudes. One can easily obtain the on-shell amplitudes of the $N \bar{N} \rightarrow P \bar{P}$ and $\Omega \bar{\Omega} \rightarrow P \bar{P}$ reactions by putting on-shell momenta of the baryons $q(t)$ and $q^{\prime}(t)$ in Eq. (D10) and

$$
k(t)=\sqrt{\frac{t}{4}-m_{P}^{2}}
$$

respectively.

The $N \bar{N}, \Omega \bar{\Omega} \rightarrow P \bar{P}$ amplitudes are calculated according to the diagram in Fig. 10. To this end, we label the two meson channels $\pi \pi, K \bar{K}$, and $\eta \eta$ as $j=1,2$, and 3, respectively, and we project the Born term, i.e., the first term in Fig. 10, into the

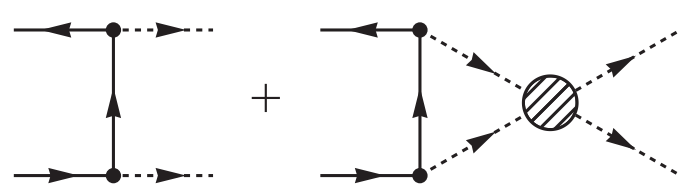

FIG. 10. Diagrammatic equation for the $N \bar{N} \rightarrow P \bar{P}$ and $\Omega \bar{\Omega} \rightarrow$ $P \bar{P}$ scattering amplitudes. Solid and dashed lines represent baryons and mesons, respectively. Shaded circle denotes the correlation of two mesons.

scalar channel as

$$
V_{j}^{(N)}(t, k, p)=\frac{1}{2} \int_{-1}^{1} d \cos \theta_{k}\left\langle j(\boldsymbol{k})|\hat{V}| N \bar{N}\left(\boldsymbol{p}, \lambda_{N}, \lambda_{N}\right)\right\rangle,
$$

$$
V_{j}^{(\Omega)}\left(t, k, p^{\prime}, \lambda_{\Omega}\right)=\frac{1}{2} \int_{-1}^{1} d \cos \theta_{k}^{\prime}\left\langle j(\boldsymbol{k})|\hat{V}| \Omega \bar{\Omega}\left(\boldsymbol{p}^{\prime}, \lambda_{\Omega}, \lambda_{\Omega}\right)\right\rangle,
$$

where $\theta_{k}^{(\prime)}$ is the angle between $\boldsymbol{p}^{(\prime)}$ and $\boldsymbol{k}$. The explicit forms of the matrix elements of $\hat{V}$ are shown in Appendix E. Next, we evaluate the diagram in Fig. 10 according to the prescription by Blankenbecler-Sugar [58] as

$$
\begin{aligned}
T_{N \bar{N} \rightarrow j}(t, k, p)= & V_{j}^{(N)}(t, k, p)+\sum_{l=1}^{3} N_{l} \int_{0}^{\infty} d k^{\prime} \frac{k^{\prime 2}}{2 \pi^{2}} \\
& \times \frac{T_{j l}^{(2 \mathrm{~m})}(t) V_{l}^{(N)}\left(t, k^{\prime}, p\right)}{\omega_{P(l)}\left(k^{\prime}\right)\left[t-4 \omega_{P(l)}\left(k^{\prime}\right)^{2}\right]}, \quad(\mathrm{D} 21) \\
T_{\Omega \bar{\Omega} \rightarrow j}\left(t, k, p^{\prime}, \lambda_{\Omega}\right)= & V_{j}^{(\Omega)}\left(t, k, p^{\prime}, \lambda_{\Omega}\right) \\
& +\sum_{l=1}^{3} N_{l} \int_{0}^{\infty} d k^{\prime} \frac{k^{\prime 2}}{2 \pi^{2}} \\
& \times \frac{T_{j l}^{(2 \mathrm{~m})}(t) V_{l}^{(\Omega)}\left(t, k^{\prime}, p^{\prime}, \lambda_{\Omega}\right)}{\omega_{P(l)}\left(k^{\prime}\right)\left[t-4 \omega_{P(l)}\left(k^{\prime}\right)^{2}\right]}, \quad(\mathrm{D} 22)
\end{aligned}
$$

where $T_{j l}^{(2 \mathrm{~m})}$ is the scalar-isoscalar $l \rightarrow j$ meson-meson scattering amplitude and $\omega_{P(l)}(k) \equiv\left(k^{2}+m_{P(l)}^{2}\right)^{1 / 2}$ with $P(l)$ being the meson in $l$ th channel. In general, $T_{j l}^{(2 \mathrm{~m})}$ should be an off-shell amplitude and thus depends on the relative momenta $k$ and $k^{\prime}$ as well. In the present study, we employ the so-called chiral unitary approach to describe $T_{j l}^{(2 \mathrm{~m})}$ together with the on-shell approximation, as explained in Appendix F, so $T_{j l}^{(2 \mathrm{~m})}$ is a function only of $t$.

With appendixes $\mathrm{E}$ and $\mathrm{F}$, all the ingredients in the above amplitudes are determined. We can check how the $N \bar{N} \rightarrow \pi \pi$ amplitude in the present formulation works by calculating the Frazer-Fulco amplitude for the $N \bar{N} \rightarrow \pi \pi$ reaction [59]

$$
f_{+}^{0}(t)=\frac{i p(t) m_{N}}{4 \sqrt{3} \pi} T_{N \bar{N} \rightarrow \pi \pi}(t, k(t), p(t)),
$$

where the factor is due to the transition to the Frazer-Fulco amplitude in isospin basis. The result is shown in Fig. 11 together with quasi-empirical values taken from Ref. [60]. 


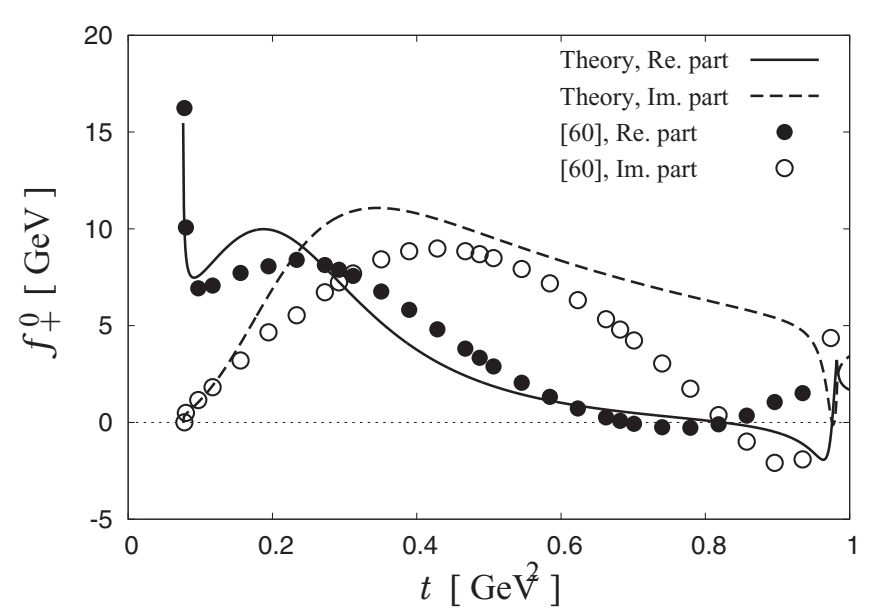

FIG. 11. Frazer-Fulco amplitude $f_{+}^{0}$ for the $N \bar{N} \rightarrow \pi \pi$ reaction. Points represent quasi-empirical values taken from Ref. [60].

The comparison indicates that our approach reproduces the quasi-empirical values semiquantitatively well.

Finally, we evaluate $\operatorname{Im} \mathcal{V}_{\mathrm{S}, 2 \mathrm{M}}(t)$ in $t>4 m_{\pi}^{2}$ from the onshell amplitudes in Eqs. (D21) and (D22) and the relation (D9). We express the right-hand side of Eq. (D9), i.e., the sum of the products of the phase space $\rho_{j}$ and amplitudes of the $j \rightarrow$ $\Omega \bar{\Omega}$ and $N \bar{N} \rightarrow j$ reactions, as $F\left(t, \lambda_{\Omega}\right)$. We note that the product $T_{\Omega \bar{\Omega} \rightarrow j}^{*} T_{N \bar{N} \rightarrow j}$ contains the uncorrelated contributions depicted as the box diagram in Fig. 12 which eventually causes the double counting in the $N \Omega$ interaction with the $\eta$ exchange term and with the box contributions of the inelastic channel in Sec. II H. We must cancel this double counting by subtracting the product of the Born terms. As a result, $F\left(t, \lambda_{\Omega}\right)$ is

$$
\begin{aligned}
F(t & \left., \lambda_{\Omega}\right) \equiv \sum_{j=1}^{3} \rho_{j}(t) \theta\left(t-m_{\mathrm{th}(j)}^{2}\right) \\
& \times\left[T_{\Omega \bar{\Omega} \rightarrow j}\left(t, k(t), p^{\prime}(t), \lambda_{\Omega}\right)^{*} T_{N \bar{N} \rightarrow j}(t, k(t), p(t))\right. \\
& \left.-V_{j}^{(\Omega)}\left(t, k(t), p^{\prime}(t), \lambda_{\Omega}\right)^{*} V_{j}^{(N)}(t, k(t), p(t))\right] . \quad(\mathrm{D} 24)
\end{aligned}
$$

With this and Eqs. (D2)-(D6), we have

$$
\begin{aligned}
& \operatorname{Im} \mathcal{V}_{\mathrm{S}}(t) \\
& =-\frac{F(t, 3 / 2)}{2 \bar{u}_{\Omega}^{\mu}\left(\boldsymbol{p}^{\prime}, 3 / 2\right) v_{\Omega} \bar{\Omega}_{\mu}\left(-\boldsymbol{p}^{\prime}, 3 / 2\right) \bar{v}_{N}(-\boldsymbol{p}, 1 / 2) u_{N}(\boldsymbol{p}, 1 / 2)} \\
& =-\frac{2 m_{N} m_{\Omega} F(t, 3 / 2)}{\sqrt{\left(4 m_{N}^{2}-t\right)\left(4 m_{\Omega}^{2}-t\right)}},
\end{aligned}
$$

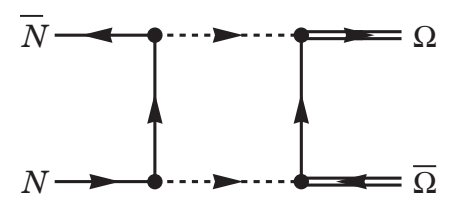

FIG. 12. Feynman box diagram for the $N \bar{N} \rightarrow \Omega \bar{\Omega}$ reaction. Solid and double lines in the intermediate state represent baryons, and dashed lines represent mesons.



FIG. 13. $\operatorname{Im} \mathcal{V}_{\mathrm{S}}$ and $\operatorname{Im} \mathcal{V}_{2 \mathrm{M}}$ in our model.

$$
\begin{aligned}
\operatorname{Im} \mathcal{V}_{2 \mathrm{M}}(t)= & \frac{m_{\Omega}^{2}}{P_{\mu} P_{\nu} \bar{u}_{\Omega}^{\mu}\left(\boldsymbol{p}^{\prime}, 1 / 2\right) v_{\Omega}^{v}\left(-\boldsymbol{p}^{\prime}, 1 / 2\right)} \\
& \times\left[-\frac{F(t, 1 / 2)}{2 \bar{v}_{N}(-\boldsymbol{p}, 1 / 2) u_{N}(\boldsymbol{p}, 1 / 2)}\right. \\
& \left.-\operatorname{Im} \mathcal{V}_{\mathrm{S}}(t) \bar{u}_{\Omega}^{\mu}\left(\boldsymbol{p}^{\prime}, 1 / 2\right) v_{\Omega \mu}\left(-\boldsymbol{p}^{\prime}, 1 / 2\right)\right] \\
= & -\frac{4 m_{N} m_{\Omega}^{3}}{\sqrt{\left(4 m_{N}^{2}-t\right)\left(4 m_{\Omega}^{2}-t\right)}\left(4 m_{\Omega}^{2}-t\right) t} \\
& \times\left[3 m_{\Omega}^{2} F(t, 1 / 2)+\left(t-3 m_{\Omega}^{2}\right) F(t, 3 / 2)\right] .
\end{aligned}
$$

In Fig. 13 we plot $\operatorname{Im} \mathcal{V}_{\mathrm{S}}$ and $\operatorname{Im} \mathcal{V}_{2 \mathrm{M}}$ in our model as functions of $t>4 m_{\pi}^{2}$. As one can see, $\operatorname{Im} \mathcal{V}_{\mathrm{S}}$ takes a non-negligible value only for $t \gtrsim 1 \mathrm{GeV}^{2}$. This may be interpreted as the exchanges of the $f_{0}(980)$ and correlated $K \bar{K}$ states. On the other hand, $\operatorname{Im} \mathcal{V}_{2 \mathrm{M}}$ has a contribution at just above the threshold $t=4 m_{\pi}^{2}$ as well, reflecting the contributions from the broad " $\sigma$ " meson and correlated $\pi \pi$.

Next, we use the dispersion relation (22) to evaluate $\mathcal{V}_{\mathrm{S}, 2 \mathrm{M}}(t)$ in $t<0$, where the correlated two-meson exchange takes place in the $N \Omega$ elastic scattering. Here we perform the integration with a cutoff $t_{c}$ instead of infinity, which we take $t_{c}=(1.2 \mathrm{GeV})^{2}$, which is the upper boundary of the fit range of our $\pi \pi-K \bar{K}-\eta \eta$ scattering amplitude in the chiral unitary approach to the experimental $\pi \pi(J=0, I=0)$ phase shift (Appendix F).

\section{APPENDIX E: EXPLICIT FORMS OF THE BORN TERMS FOR THE $N \bar{N}, \Omega \bar{\Omega} \rightarrow$ MESON-MESON REACTIONS}

Here we show the explicit forms of the Born terms for the $N\left(\boldsymbol{p}, \lambda_{N}\right) \bar{N}\left(-\boldsymbol{p}, \lambda_{N}\right) \rightarrow P(\boldsymbol{k}) \bar{P}(-\boldsymbol{k})$ and $\Omega\left(\boldsymbol{p}^{\prime}, \lambda_{\Omega}\right) \bar{\Omega}\left(-\boldsymbol{p}^{\prime}, \lambda_{\Omega}\right) \rightarrow P(\boldsymbol{k}) \bar{P}(-\boldsymbol{k}) \quad$ reactions, where $P \bar{P}=\pi \pi, K \bar{K}$, and $\eta \eta$. Here $\boldsymbol{p}, \boldsymbol{p}^{\prime}$, and $\boldsymbol{k}$ are the relative momenta of $N \bar{N}, \Omega \bar{\Omega}$, and $P \bar{P}$, respectively, for which we take, without loss of generality, $\boldsymbol{p}=(0,0, p), \boldsymbol{p}^{\prime}=\left(0,0, p^{\prime}\right)$, and $\boldsymbol{k}=(k \sin \theta, 0, k \cos \theta)$ throughout this section. We 
define four-momenta $p_{N}^{\mu}=(\sqrt{t} / 2, \boldsymbol{p}), p_{\bar{N}}^{\mu}=(\sqrt{t} / 2,-\boldsymbol{p})$, $p_{\Omega}^{\mu}=\left(\sqrt{t} / 2, \boldsymbol{p}^{\prime}\right), p_{\bar{\Omega}}^{\mu}=\left(\sqrt{t} / 2,-\boldsymbol{p}^{\prime}\right), k_{P}^{\mu} \equiv k^{\mu}=(\sqrt{t} / 2, \boldsymbol{k})$, and $k_{\bar{P}}^{\mu} \equiv k^{\prime \mu}=(\sqrt{t} / 2,-\boldsymbol{k})$. Helicities of antiparticles are constrained as $\lambda_{\bar{N}}=\lambda_{N}$ and $\lambda_{\bar{\Omega}}=\lambda_{\Omega}$ so as to construct $J=0$.

\section{1. $N \bar{N} \rightarrow$ meson-meson}

First, the $N \bar{N} \rightarrow \pi \pi, K \bar{K}$, and $\eta \eta$ Born terms are calculated as

$$
\begin{aligned}
\left\langle\pi \pi(\boldsymbol{k})|\hat{V}| N \bar{N}\left(\boldsymbol{p}, \lambda_{N}, \lambda_{N}\right)\right\rangle & =\mathcal{F}_{\pi N N} \mathcal{V}_{N N N}+\mathcal{F}_{\pi N \Delta} \mathcal{V}_{N \Delta N}+(\boldsymbol{k} \leftrightarrow-\boldsymbol{k}), \\
\left\langle K \bar{K}(\boldsymbol{k})|\hat{V}| N \bar{N}\left(\boldsymbol{p}, \lambda_{N}, \lambda_{N}\right)\right\rangle & =\mathcal{F}_{K N \Lambda} \mathcal{V}_{N \Lambda N}+\mathcal{F}_{K N \Sigma} \mathcal{V}_{N \Sigma N}+\mathcal{F}_{K N \Sigma^{*}} \mathcal{V}_{N \Sigma^{*} N}, \\
\left\langle\eta \eta(\boldsymbol{k})|\hat{V}| N \bar{N}\left(\boldsymbol{p}, \lambda_{N}, \lambda_{N}\right)\right\rangle & =\mathcal{F}_{\eta N N} \mathcal{V}_{N N N}+(\boldsymbol{k} \leftrightarrow-\boldsymbol{k})
\end{aligned}
$$

The notation $(\boldsymbol{k} \leftrightarrow-\boldsymbol{k})$ means to add the symmetrized contributions for the identical two-meson systems. Here $\mathcal{F}_{P B B}$ and $\mathcal{F}_{P B D}$ are coupling constants which are given by

$$
\begin{gathered}
\mathcal{F}_{\pi N N}=-\frac{\sqrt{3}(D+F)^{2}}{4 f_{\pi}^{2}}, \quad \mathcal{F}_{\pi N \Delta}=-\frac{2}{\sqrt{3}} \frac{f_{P B D}^{2}}{m_{\pi}^{2}}, \\
\mathcal{F}_{K N \Lambda}=-\frac{(D+3 F)^{2}}{12 \sqrt{2} f_{K}^{2}}, \quad \mathcal{F}_{K N \Sigma}=-\frac{3(D-F)^{2}}{4 \sqrt{2} f_{K}^{2}}, \\
\mathcal{F}_{K N \Sigma^{*}}=-\frac{1}{2 \sqrt{2}} \frac{f_{P B D}^{2}}{m_{\pi}^{2}}, \quad \mathcal{F}_{\eta N N}=\frac{(D-3 F)^{2}}{12 f_{\eta}^{2}} .
\end{gathered}
$$

Terms $\mathcal{V}_{N B N}$ and $\mathcal{V}_{N D N}$ are the amplitudes of the octet- and decuplet-baryon exchange for the $N \bar{N}$ scattering, respectively, as functions of $t, \boldsymbol{k}$, and $\boldsymbol{p}$ :

$$
\begin{aligned}
\mathcal{V}_{N B N}(t, \boldsymbol{k}, \boldsymbol{p})= & -F(k)^{2} \bar{v}_{N}(-\boldsymbol{p},+1 / 2) k^{\prime} \gamma_{5} S_{B}\left(p_{N}-k\right) \\
& \times k \gamma_{5} u_{N}(\boldsymbol{p},+1 / 2),
\end{aligned}
$$

where $S_{B}(p)$ is the propagator of the octet baryon $B$

$$
S_{B}(p) \equiv \frac{\not p+m_{B}}{\left(p^{\mu}\right)^{2}-m_{B}^{2}},
$$

with its mass $m_{B}$, and

$$
\begin{aligned}
\mathcal{V}_{N D N}\left(t, \boldsymbol{k}, \boldsymbol{p}, \lambda_{N}\right)= & F(k)^{2} \bar{v}_{N}(-\boldsymbol{p},+1 / 2) k_{\mu}^{\prime} S_{D}^{\mu \nu}\left(p_{N}-k\right) \\
& \times k_{\nu} u_{N}(\boldsymbol{p},+1 / 2),
\end{aligned}
$$

where $S_{D}(p)$ is the propagator of the decuplet baryon $D$

$$
\begin{aligned}
S_{D}^{\mu \nu}(p)= & \frac{\not p+m_{D}}{\left(p^{\mu}\right)^{2}-m_{D}^{2}}\left[g^{\mu \nu}-\frac{1}{3} \gamma^{\mu} \gamma^{\nu}\right. \\
& \left.-\frac{2 p^{\mu} p^{\nu}}{3 m_{D}^{2}}+\frac{p^{\mu} \gamma^{\nu}-p^{\nu} \gamma^{\mu}}{3 m_{D}}\right],
\end{aligned}
$$

with its mass $m_{D}$. As for the form factor $F(k)$ in the $N \bar{N}$, $\Omega \Omega \rightarrow P \bar{P}$ amplitudes, we employ the monopole type in Eq. (15) and use the same value of the cutoff $\Lambda=1 \mathrm{GeV}$. We do not include the width of the decuplet baryons in the propagator. Note that neither $\mathcal{V}_{N B N}$ nor $\mathcal{V}_{N D N}$ depend on the helicity $\lambda_{N}$, so we take $\lambda_{N}=+1 / 2$ here.

\section{2. $\Omega \bar{\Omega} \rightarrow$ meson-meson}

Next, the $\Omega \bar{\Omega} \rightarrow \pi \pi, K \bar{K}$, and $\eta \eta$ Born terms are calculated as

$$
\left\langle\pi \pi(\boldsymbol{k})|\hat{V}| \Omega \bar{\Omega}\left(\boldsymbol{p}^{\prime}, \lambda_{\Omega}, \lambda_{\Omega}\right)\right\rangle=0,
$$

$$
\left\langle K \bar{K}(\boldsymbol{k})|\hat{V}| \Omega \bar{\Omega}\left(\boldsymbol{p}^{\prime}, \lambda_{\Omega}, \lambda_{\Omega}\right)\right\rangle=\mathcal{F}_{K \Xi \Omega} \mathcal{V}_{\Omega \Xi \Omega}+\mathcal{F}_{K \Xi^{*} \Omega} \mathcal{V}_{\Omega \Xi^{*} \Omega},
$$

$$
\left\langle\eta \eta(\boldsymbol{k})|\hat{V}| \Omega \bar{\Omega}\left(\boldsymbol{p}^{\prime}, \lambda_{\Omega}, \lambda_{\Omega}\right)\right\rangle=\mathcal{F}_{\eta \Omega \Omega} \mathcal{V}_{\Omega \Omega \Omega}+(\boldsymbol{k} \leftrightarrow-\boldsymbol{k}) .
$$

Here $\mathcal{F}_{P B B}$ and $\mathcal{F}_{P B D}$ are coupling constants defined as

$$
\begin{aligned}
\mathcal{F}_{K \Xi \Omega} & =\sqrt{2} \frac{f_{P B D}^{2}}{m_{\pi}^{2}}, \quad \mathcal{F}_{K \Xi * \Omega}=-\frac{\sqrt{2} f_{P D D}^{2}}{3 m_{\pi}^{2}}, \\
\mathcal{F}_{\eta \Omega \Omega} & =\frac{2 f_{P D D}}{3 m_{\pi}^{2}} .
\end{aligned}
$$

Terms $\mathcal{V}_{\Omega B \Omega}$ and $\mathcal{V}_{\Omega D \Omega}$ are the amplitudes of the octet- and decuplet-baryon exchange for the $\Omega \bar{\Omega}$ scattering, respectively, as functions of $t, \boldsymbol{k}, \boldsymbol{p}^{\prime}$, and $\lambda_{N}$ :

$$
\begin{aligned}
\mathcal{V}_{\Omega B \Omega}\left(t, \boldsymbol{k}, \boldsymbol{p}^{\prime}, \lambda_{\Omega}\right)= & F(k)^{2} \bar{v}_{\Omega}^{\mu}\left(-\boldsymbol{p}^{\prime}, \lambda_{\Omega}\right) k_{\mu}^{\prime} S_{B}\left(p_{\Omega}-k\right) \\
& \times k_{v} u_{\Omega}^{v}\left(\boldsymbol{p}^{\prime}, \lambda_{\Omega}\right), \\
\mathcal{V}_{\Omega D \Omega}\left(t, \boldsymbol{k}, \boldsymbol{p}^{\prime}, \lambda_{\Omega}\right)= & F(k)^{2} \bar{v}_{\Omega \mu}\left(-\boldsymbol{p}^{\prime}, \lambda_{\Omega}\right) k^{\prime} \gamma_{5} S_{D}^{\mu \nu}\left(p_{\Omega}-k\right) \\
& \times k \gamma_{5} u_{\Omega v}\left(\boldsymbol{p}^{\prime}, \lambda_{\Omega}\right),
\end{aligned}
$$

Note that the amplitudes depend on the helicity $\lambda_{\Omega}$ but this dependence will be canceled when divided by the bispinor $\bar{u}_{\Omega}^{\mu}\left(\boldsymbol{p}^{\prime}, \lambda_{\Omega}\right) v_{\bar{\Omega} \mu}\left(-\boldsymbol{p}^{\prime}, \lambda_{\Omega}\right)$ as in Eq. (D9).

\section{APPENDIX F: MESON-MESON SCATTERING AMPLITUDE}

In this study we describe the $\pi \pi-K \bar{K}-\eta \eta$ coupled-channels scattering amplitude in the scalar-isoscalar channel by using the so-called chiral unitary approach [38-41]. In this approach we calculate the scattering amplitude of two pseudoscalar mesons $T_{j k}^{(2 \mathrm{~m})}(s)$, where $j$ and $k$ are channel indices $(\pi \pi$, $K \bar{K}$, and $\eta \eta$ for $j=1,2$, and 3, respectively) and $s$ is the Mandelstam variable, by solving the Lippmann-Schwinger equation in the following form:

$$
T_{j k}^{(2 \mathrm{~m})}(s)=V_{j k}^{(2 \mathrm{~m})}(s)+\sum_{l} V_{j l}^{(2 \mathrm{~m})}(s) G_{l}^{(2 \mathrm{~m})}(s) T_{l k}^{(2 \mathrm{~m})}(s),
$$

with the interaction kernel $V_{j k}=V_{k j}$ taken from chiral perturbation theory and the loop function of two pseudoscalar mesons $G_{j}$. 
We employ the leading-order terms of chiral perturbation theory for the interaction kernel $V_{j k}$ :

$$
\begin{aligned}
& V_{11}^{(2 \mathrm{~m})}(s)=\frac{m_{\pi}^{2}-2 s}{f_{\pi}^{2}}, \quad V_{12}^{(2 \mathrm{~m})}(s)=-\frac{\sqrt{3} s}{2 \sqrt{2} f_{\pi} f_{K}}, \\
& V_{13}^{(2 \mathrm{~m})}(s)=\frac{m_{\pi}^{2}}{\sqrt{3} f_{\pi} f_{\eta}}, \quad V_{22}^{(2 \mathrm{~m})}(s)=-\frac{3 s}{4 f_{K}^{2}}, \\
& V_{23}^{(2 \mathrm{~m})}(s)=\frac{9 s-2 m_{\pi}^{2}-6 m_{\eta}^{2}}{6 \sqrt{2} f_{K} f_{\pi}}, \\
& V_{33}^{(2 \mathrm{~m})}(s)=\frac{7 m_{\pi}^{2}-16 m_{K}^{2}}{9 f_{\eta}^{2}},
\end{aligned}
$$

where the on-shell approximation was used. The loop function $G_{j}$ is evaluated with a three-dimensional sharp cutoff $q_{\max }$ :

$$
\begin{aligned}
G_{j}^{(2 \mathrm{~m})}(s) & =i N_{j} \int \frac{d^{4} q}{(2 \pi)^{4}} \frac{1}{\left(q^{2}-m_{j}^{2}\right)\left[(P-q)^{2}-m_{j}^{2}\right]} \\
& =\frac{N_{j}}{2 \pi^{2}} \int_{0}^{q_{\max }} d q \frac{q^{2}}{\omega_{j}(q)\left[s-4 \omega_{j}(q)^{2}\right]}
\end{aligned}
$$

where $m_{j}$ is the meson mass in $j$ th channel, $P^{\mu}=(\sqrt{s}, \mathbf{0})$,

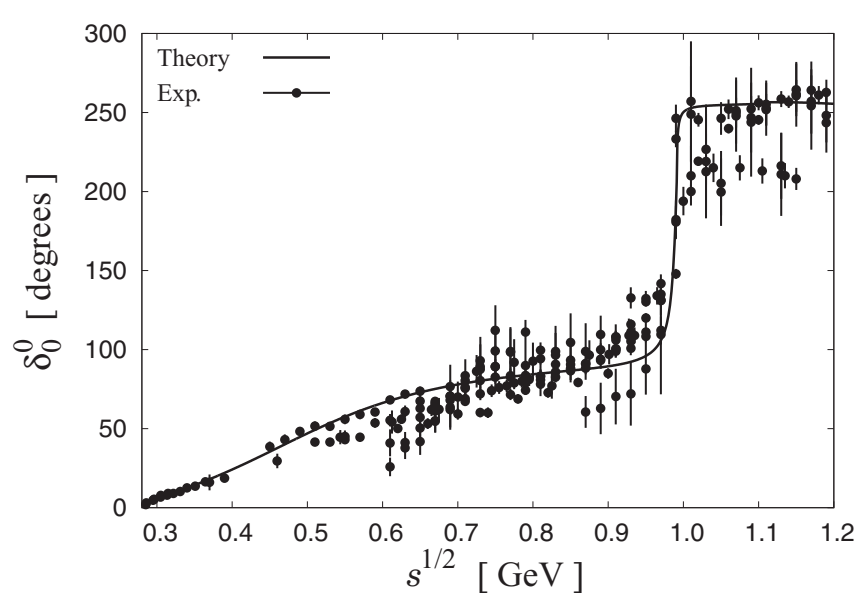

FIG. 14. $\pi \pi(J=0, I=0)$ phase shift $\delta_{0}^{0}$ in the present model. Experimental data are taken from Refs. [61-65].

$\omega_{j}(q) \equiv\left(q^{2}+m_{j}^{2}\right)^{1 / 2}$, and the symmetry factor is $N_{1}=N_{3}=$ $1 / 2$ and $N_{2}=1$. In this construction only the cutoff $q_{\max }$ is the model parameter, and we fix it by fitting the $\pi \pi(J=0, I=0)$ scattering phase shift $\delta_{0}^{0}$ to the experimental data [61-65] up to $\sqrt{s}=1.2 \mathrm{GeV}$. From the best fit, we use the value of the cutoff $q_{\max }=850 \mathrm{MeV}$, with which we can reproduce the phase shift $\delta_{0}^{0}$ fairly well as shown in Fig. 14.
[1] S. Weinberg, Phys. Rev. 137, B672 (1965).

[2] F. Dyson and N. H. Xuong, Phys. Rev. Lett. 13, 815 (1964).

[3] R. L. Jaffe, Phys. Rev. Lett. 38, 195 (1977); 38, 617 (1977).

[4] H. Clement, Prog. Part. Nucl. Phys. 93, 195 (2017).

[5] T. Yamazaki and Y. Akaishi, Phys. Lett. B 535, 70 (2002).

[6] Y. Akaishi and T. Yamazaki, Phys. Rev. C 65, 044005 (2002).

[7] T. Hyodo and D. Jido, Prog. Part. Nucl. Phys. 67, 55 (2012).

[8] A. Gal, E. V. Hungerford, and D. J. Millener, Rev. Mod. Phys. 88, 035004 (2016).

[9] M. Bashkanov et al. (CELSIUS/WASA Collaboration), Phys. Rev. Lett. 102, 052301 (2009).

[10] P. Adlarson et al. (WASA-at-COSY Collaboration), Phys. Rev. Lett. 106, 242302 (2011).

[11] P. Adlarson et al. (WASA-at-COSY Collaboration), Phys. Lett. B 721, 229 (2013).

[12] S. R. Beane, E. Chang, W. Detmold, B. Joo, H. W. Lin, T. C. Luu, K. Orginos, A. Parreño, M. J. Savage, A. Torok, and A. Walker-Loud (NPLQCD Collaboration), Phys. Rev. Lett. 106, 162001 (2011).

[13] T. Inoue, N. Ishii, S. Aoki, T. Doi, T. Hatsuda, Y. Ikeda, K. Murano, H. Nemura, and K. Sasaki (HAL QCD Collaboration), Phys. Rev. Lett. 106, 162002 (2011).

[14] S. R. Beane, E. Chang, W. Detmold, H. W. Lin, T. C. Luu, K. Orginos, A. Parreno, M. J. Savage, A. Torok, and A. WalkerLoud (NPLQCD Collaboration), Phys. Rev. D 85, 054511 (2012).

[15] T. Inoue et al. (HAL QCD Collaboration), Nucl. Phys. A 881, 28 (2012).

[16] K. Sasaki et al. (HAL QCD Collaboration), Nucl. Phys. A 914, 231 (2013).
[17] K. Sasaki et al. (HAL QCD Collaboration), Prog. Theor. Exp. Phys. 2015, 113B01 (2015).

[18] K. Sasaki et al., PoS LATTICE 2015, 088 (2016).

[19] K. Sasaki et al., PoS LATTICE 2016, 116 (2017).

[20] S. Gongyo et al. (HAL QCD Collaboration), Phys. Rev. Lett. 120, 212001 (2018).

[21] Y. Sada et al. (J-PARC E15 Collaboration), Prog. Theor. Exp. Phys. 2016, $051 \mathrm{D} 01$ (2016).

[22] T. Sekihara, E. Oset, and A. Ramos, Prog. Theor. Exp. Phys. 2016, 123D03 (2016).

[23] T. Goldman, K. Maltman, G. J. Stephenson, Jr., K. E. Schmidt, and F. Wang, Phys. Rev. Lett. 59, 627 (1987).

[24] M. Oka, Phys. Rev. D 38, 298 (1988).

[25] Q. B. Li and P. N. Shen, Eur. Phys. J. A 8, 417 (2000).

[26] H. Pang, J. Ping, F. Wang, J. Goldman, and E. Zhao, Phys. Rev. C 69, 065207 (2004).

[27] X. Zhu, H. Huang, J. Ping, and F. Wang, Phys. Rev. C 92, 035210 (2015).

[28] H. Huang, J. Ping, and F. Wang, Phys. Rev. C 92, 065202 (2015).

[29] F. Etminan et al. (HAL QCD Collaboration), Nucl. Phys. A 928, 89 (2014).

[30] T. Doi et al., EPJ Web Conf. 175, 05009 (2018).

[31] T. Iritani et al. (HAL QCD Collaboration) (unpublished).

[32] J. Haidenbauer, S. Petschauer, N. Kaiser, U. G. Meißner, and W. Weise, Eur. Phys. J. C 77, 760 (2017).

[33] K. Morita, A. Ohnishi, F. Etminan, and T. Hatsuda, Phys. Rev. C 94, 031901 (2016).

[34] C. Patrignani (Particle Data Group Collaboration), Chin. Phys. C 40, 100001 (2016).

[35] G. E. Brown and W. Weise, Phys. Rep. 22, 279 (1975). 
[36] H. C. Kim, J. W. Durso, and K. Holinde, Phys. Rev. C 49, 2355 (1994).

[37] A. Reuber, K. Holinde, H. C. Kim, and J. Speth, Nucl. Phys. A 608, 243 (1996).

[38] J. A. Oller and E. Oset, Nucl. Phys. A 620, 438 (1997); 652, 407 (1999).

[39] J. A. Oller, E. Oset, and J. R. Pelaez, Phys. Rev. Lett. 80, 3452 (1998).

[40] J. A. Oller, E. Oset, and J. R. Pelaez, Phys. Rev. D 59, 074001 (1999); 60, 099906 (1999); 75, 099903 (2007).

[41] J. A. Oller and E. Oset, Phys. Rev. D 60, 074023 (1999).

[42] T. Iritani et al. (HAL QCD Collaboration) (private communication).

[43] K.-I. Ishikawa et al. (PACS Collaboration), PoS LATTICE 2015, 075 (2016).

[44] V. Baru, J. Haidenbauer, C. Hanhart, Y. Kalashnikova, and A. E. Kudryavtsev, Phys. Lett. B 586, 53 (2004).

[45] T. Sekihara, T. Hyodo, and D. Jido, Prog. Theor. Exp. Phys. 2015, 063 D04 (2015).

[46] T. Sekihara and S. Kumano, Phys. Rev. D 92, 034010 (2015).

[47] T. Sekihara, Phys. Rev. C 95, 025206 (2017).

[48] T. Hyodo, D. Jido, and A. Hosaka, Phys. Rev. C 85, 015201 (2012).

[49] T. Hyodo, Int. J. Mod. Phys. A 28, 1330045 (2013).

[50] J. Formánek, R. J. Lombard, and J. Mareš, Czech. J. Phys. B 54, 289 (2004).
[51] K. Miyahara and T. Hyodo, Phys. Rev. C 93, 015201 (2016).

[52] Y. Kamiya and T. Hyodo, Phys. Rev. C 93, 035203 (2016).

[53] Y. Kamiya and T. Hyodo, Prog. Theor. Exp. Phys. 2017, 023 D02 (2017).

[54] S. Cho, T. Furumoto, T. Hyodo, D. Jido, C. M. Ko, S. H. Lee, M. Nielsen, A. Ohnishi, T. Sekihara, S. Yasui, and K. Yazaki (ExHIC Collaboration), Phys. Rev. Lett. 106, 212001 (2011).

[55] S. Cho, T. Furumoto, T. Hyodo, D. Jido, C. M. Ko, S. H. Lee, M. Nielsen, A. Ohnishi, T. Sekihara, S. Yasui, and K. Yazaki (ExHIC Collaboration), Phys. Rev. C 84, 064910 (2011).

[56] S. Cho et al. (ExHIC Collaboration), Prog. Part. Nucl. Phys. 95, 279 (2017).

[57] M. Jacob and G. C. Wick, Ann. Phys. 7, 404 (1959); 281, 774 (2000).

[58] R. Blankenbecler and R. Sugar, Phys. Rev. 142, 1051 (1966).

[59] W. R. Frazer and J. R. Fulco, Phys. Rev. 117, 1603 (1960).

[60] O. Dumbrajs, R. Koch, H. Pilkuhn, G. c. Oades, H. Behrens, J. j. De Swart, and P. Kroll, Nucl. Phys. B 216, 277 (1983).

[61] S. D. Protopopescu et al., Phys. Rev. D 7, 1279 (1973).

[62] G. Grayer et al., Nucl. Phys. B 75, 189 (1974).

[63] K. N. Mukhin, O. O. Patarakin, M. M. Sulkovskaya, L. V. Surkova, and A. F. Sustavov, Pis'ma Zh. Eksp. Teor. Fiz. 32, 616 (1980) [JETP Lett. 32, 601 (1980)].

[64] R. Kaminski, L. Lesniak, and K. Rybicki, Eur. Phys. J. direct 4, 1 (2002).

[65] J. R. Batley et al. (NA48-2 Collaboration), Eur. Phys. J. C 70, 635 (2010). 